\title{
ARTICLE
}

\section{UFMylation of RPL26 links translocation-associated quality control to endoplasmic reticulum protein homeostasis}

\author{
Lihui Wang ${ }^{1}$, Yue $\mathrm{Xu}^{1}$, Heather Rogers ${ }^{2}$, Layla Saidi ${ }^{1}$, Constance Tom Noguchi ${ }^{2}$, Honglin $\mathrm{Li}^{3}$, Jonathan Wilson Yewdell ${ }^{4}$, \\ Nicholas Raymond Guydosh ${ }^{5}$ and Yihong Ye ${ }^{1}$
}

Protein biogenesis at the endoplasmic reticulum (ER) in eukaryotic cells is monitored by a protein quality control system named ERassociated protein degradation (ERAD). While there has been substantial progress in understanding how ERAD eliminates defective polypeptides generated from erroneous folding, how cells remove nascent chains stalled in the translocon during co-translational protein insertion into the ER is unclear. Here we show that ribosome stalling during protein translocation induces the attachment of UFM1, a ubiquitin-like modifier, to two conserved lysine residues near the COOH-terminus of the 60S ribosomal subunit RPL26 (uL24) at the ER. Strikingly, RPL26 UFMylation enables the degradation of stalled nascent chains, but unlike ERAD or previously established cytosolic ribosome-associated quality control (RQC), which uses proteasome to degrade their client proteins, ribosome UFMylation promotes the targeting of a translocation-arrested ER protein to lysosomes for degradation. RPL26 UFMylation is upregulated during erythroid differentiation to cope with increased secretory flow, and compromising UFMylation impairs protein secretion, and ultimately hemoglobin production. We propose that in metazoan, co-translational protein translocation into the ER is safeguarded by a UFMylation-dependent protein quality control mechanism, which when impaired causes anemia in mice and abnormal neuronal development in humans.

Cell Research (2020) 30:5-20; https://doi.org/10.1038/s41422-019-0236-6

\section{INTRODUCTION}

To maintain protein homeostasis in the endoplasmic reticulum (ER), a secretory compartment responsible for the production of approximately one-third of the mammalian proteins, eukaryotic cells have evolved a protein quality control system termed ERassociated protein degradation (ERAD), which exports malfolded polypeptides from the ER into the cytosol for degradation by proteasome. ${ }^{1-3}$ Although significant advance has been made in understanding the role of ERAD in degradation of defective proteins generated from erroneous folding post ER delivery, little is known about how cells eliminate polypeptides stalled in the translocon during co-translational targeting, a scenario that can occur when ribosome translation is arrested on mRNAs encoding ER-bound proteins.

In eukaryotic cells, a major source of erroneous translation that causes ribosome stalling comes from naturally occurring faulty mRNAs. For instance, non-stop mRNAs bearing polyadenine (polyA) stretch are generated in as much as $5 \%-10 \%$ transcripts in eukaryotic cells due to premature polyadenylation. ${ }^{4}$ Translation of poly-A stretches that are normally outside of open reading frames (ORFs) can also cause ribosome stalling or prolonged pausing, resulting in arrested products (AP) that are prone to misfolding and aggregation. These aberrant translation products can interfere with other functional proteins and are therefore deleterious to cells. In the cytosol, a ribosome-associated quality control (RQC) pathway effectively eliminates APs upon ribosome stalling. ${ }^{5,6}$ This RQC pathway employs a collection of factors that coordinately sense translation stalling, rescue stalled ribosomes, and degrade aberrant nascent polypeptides. ${ }^{7}$ Specifically, ubiquitination of $40 \mathrm{~S}$ ribosomal subunits by the ribosome-associated ubiquitin ligase (E3) ZNF598 serves as a signal to trigger ribosome splitting by a ribosome recycling factor (e.g., PELO and ABCE1). ${ }^{8-12}$ Subsequently, the 605 ribosome-associated ubiquitin ligase Listerin and its binding partner NEMF are recruited, resulting in AP ubiquitination and degradation by proteasome. ${ }^{13-17}$ RQC deficiency can cause abnormal development and neurodegeneration in animals, ${ }^{15,18-22}$ underscoring the importance of this quality control pathway in protein homeostasis regulation.

In sharp contrast to the cytosolic RQC pathway, little is known about the quality control mechanism for endoplasmic reticulum (ER)-bound ribosomes when they encounter translation arrest. Unlike ribosome stalling in the cytosol, translation arrest during co-translational protein translocation at the ER creates a unique "crisis" because APs are situated inside the Sec61 translocon with their N-termini in the ER lumen, which may have participated in protein biogenesis-associated events such as folding, glycosylation, and disulfide bond formation. Thus, the removal of translation-arrested ER proteins might engage a mechanism more

\footnotetext{
${ }^{1}$ Laboratory of Molecular Biology, National Institute of Diabetes and Digestive and Kidney Diseases, National Institutes of Health, Bethesda, MD 20892, USA; ${ }^{2}$ Molecular Medicine Branch, National Institute of Diabetes and Digestive and Kidney Diseases, National Institutes of Health, Bethesda, MD 20892, USA; ${ }^{3}$ Department of Biochemistry and Molecular Biology, Augusta University Medical Center, Augusta, GA 30912, USA; ${ }^{4}$ Laboratory of Viral Diseases, National Institute of Allergy and Infectious Diseases, National Institutes of Health, Bethesda, MD 20892, USA and ${ }^{5}$ Laboratory of Biochemistry and Genetics, National Institute of Diabetes and Digestive and Kidney Diseases, National Institutes of Health, Bethesda, MD 20892, USA

Correspondence: Yihong Ye (yihongy@mail.nih.gov)
}

Received: 7 May 2019 Accepted: 6 September 2019

Published online: 8 October 2019 
akin to ERAD than cytosolic RQC. Failure to degrade nascent chains stalled in the translocon is expected to cause accumulation of APs in the ER, which could interfere with ER protein biogenesis, leading to a secretion defect and ER stress.

UFM1 is a small ubiquitin-like modifier essential for cell differentiation and animal development. ${ }^{23-26}$ Mutations in genes encoding human UFMylation enzymes are linked to early onset of encephalopathy ${ }^{27-29}$ and cerebellar ataxia, ${ }^{30}$ while mice lacking the functional UFMylation system have multiple defects during embryonic development including a severe deficiency in erythroid differentiation. ${ }^{24-26}$ However, the properties of the few candidate UFMylation substrates reported to date (ASC1, UFBP1, and histone $\mathrm{H} 4)^{31-33}$ do not readily explain these phenotypes. Along this line, a recent study reported an interaction between the UFM1 ligase UFL 1 and the 80 s ribosome, ${ }^{34}$ while another study identified the ribosome large subunit RPL26 as a UFMylation substrate. ${ }^{35}$ The position of RPL26 in ribosomes translating ER-bound nascent chains suggests a possible role for ribosome UFMylation in ER protein biogenesis, but the precise function of ribosome UFMylation is unclear.

In this study, we independently confirm RPL26 as a primary substrate of UFMylation in mammalian cells and identify two conserved lysine residues in the C-terminal tail of RPL26 as the major UFMylation sites. Importantly, our study reveals translation arrest during co-translational protein translocation as a specific regulator of ribosome UFMylation. RPL26 UFMylation enables efficient elimination of translocation-stalled polypeptides, but surprisingly, this is not mediated by the canonical ERAD system or previously established cytosolic RQC. Instead, translocation-stalled ER-nascent chains can be transported to lysosomes for destruction. Our findings collectively define an unconventional branch of ERAD that links protein translocation-associated quality control to erythroid differentiation and neuronal development.

\section{RESULTS}

RPL26 is UFMylated at two conserved lysine residues in the Cterminal tail

To understand the substrate scope and biological relevance of protein UFMylation, we immunoblotted whole cell extracts from a panel of mammalian cell lines with UFM1-specific antibodies. Surprisingly, this revealed only two major protein bands in all cell lines (these UFMylation products are designated as "S1" and "S2" hereafter) (Supplementary information, Fig. S1a). Both S1 and S2 were specific UFMylation products because CRISPR-mediated inactivation of UFL1, a component of the sole UFM1 ligase (E3) complex ${ }^{24,36}$ reduced their levels and inactivation of the deufmylase UFSP2 ${ }^{37}$ had the opposite effect (Supplementary information, Fig. S1b, c). Like ubiquitination, modification of the C-terminus of UFM1 (removal of the three C-terminal residues, generating " $\Delta C 3^{\prime \prime}$ ) abrogated the S1 and S2 formation (Supplementary information, Fig. S1d, e). Biochemical fractionation showed that S1 and S2 co-sedimented with the ER membranes (Supplementary information, Fig. S1g, h), consistent with the ER localization of the E3 components UFL1 and UFBP1 ${ }^{38}$ (Supplementary information, Fig. S1f).

To identify UFMylation substrate(s), we affinity-purified FLAGtagged wild-type (WT) UFM1 or UFM1 $\triangle$ C3 under denaturing conditions from membrane fractions of UFSP2 CRISPR knockout (KO) cells (Supplementary information, Fig. S2a). This confirmed S1 UFMylation since S1 was only recovered from cells expressing FLAG-tagged WT UFM1 but not UFM1 $\triangle$ C3 (Fig. 1a). S2, due to its low abundance, was not recovered to a level detectable by silver staining. Mass spectrometry analysis revealed that S1 consists of two proteins: UFM1 and RPL26 (Fig. 1b, Supplementary information, Table S1).

To confirm RPL26 as a UFMylation substrate, we expressed FLAG-tagged RPL26 or RPL26-like protein-1 (RPL26L1) (the latter differs from RPL26 by two amino acids) in cells. We also examined RPL10-FLAG, a reported UFMylation substrate in mouse embryonic stem cells. $^{34}$ Ribosome purification using sucrose cushion confirmed that these proteins were all incorporated into ribosomes (Supplementary information, Fig. S2b). Immunoprecipitation with FLAG antibodies (FLAG-IP) followed by immunoblotting with UFM1 antibodies showed that RPL26 and RPL26L1 both yielded species corresponding to $\mathrm{S} 1$ and $\mathrm{S} 2$ with $\mathrm{S} 1$ being the predominant species (Fig. 1c). This suggested that S1 and S2 both represent UFMylated RPL26, which based on their molecular mass (Mr), contain, respectively, one and two attached UFM1 moieties. Immunoblotting with FLAG antibodies further confirmed that a small fraction of RPL26 is modified at steady state (Fig. 1c). However, we failed to detect a UFM1-positive signal with RPL10.

To test UFMylation of endogenous RPL26, we immunoblotted ribosomes purified from UFSP2 KO cells by sucrose cushion. The result showed that endogenous $\mathrm{S} 1$ was recognized by both UFM1and RPL26-specific antibodies, whereas S2, likely due to its lower abundance, was only detected by UFM1 antibody (Fig. 1d). UFMylation of endogenous RPL26 was similarly observed when membranes isolated from semi-permeabilized HEK293T cells were incubated with purified UBA5 (E1), UFC1 (E2), UFM1 and ATP (Supplementary information, Fig. S2c). Upon ribosome fractionation, both S1 and S2 were predominantly detected in the ribosome fractions (Fig. 1d). Preventing RPL26 incorporation into ribosomes by FLAG tagging at its amino terminus (which is buried in ribosomes) abrogated UFMylation (Supplementary information, Fig. S2d, e, Fig. 1e). Thus, RPL26 UFMylation only occurs when RPL26 is incorporated into ribosomes.

Sequence alignment showed that human RPL26 contains a 19-residue $\mathrm{COOH}$-tail that is present in metazoan, but not in fungal RPL26 paralogues (Fig. 1f). Since the UFMylation system is absent in fungi, this suggests that metazoan RPL26 $\mathrm{COOH}$-tail evolution was sculpted by the UFMylation system. Consistent with this notion, UFMylation of a tail-deleted RPL26 mutant transiently expressed in HEK293T cells was reduced by $\sim 80 \%$ (Fig. 1g). Furthermore, CRISPR-mediated gene editing to replace the endogenous RPL26 COOH-tail in one allele with an irrelevant peptide (Supplementary information, Fig. S3a-c) reduced S1 and S2 levels by $\sim 50 \%$ in K562 cells (Fig. 1h). Editing both copies in HEK293T cells completely abolished RPL26 UFMylation (Supplementary information, Fig. S3d, e). Because deleting RPL26 C-tail did not affect ribosome integrity (Supplementary information, Fig. S3f, g), we conclude that S1 and S2 are generated by UFMylation of the RPL26 $\mathrm{COOH}$-tail.

The RPL26 COOH-tail contains several lysine residues with two being highly conserved (K132 and K134) (Fig. 1f). Substitution of K132 to arginine (R) almost completely abolished S2 but did not affect S1 (Supplementary information, Fig. S4a), whereas the K134R mutation reduced S1 and S2 by $\sim 80 \%$ and $\sim 50 \%$, respectively. When these mutations were combined, both $\mathrm{S} 1$ and S2 were significantly reduced (Supplementary information, Fig. S4b). Collectively, these results suggested that S1 is formed mostly by UFMylation of RPL26 at Lys134, whereas S2 is a result of combined modification at Lys132 together with either Lys134 or a yet-to-be identified site (Supplementary information, Fig. S4c). Importantly, both Lys132 and Lys134 are solvent-exposed and proximal to the polypeptide exit tunnel that should face the ER membrane for ribosomes translating ER-bound proteins (Fig. 1e).

Ribosome stalling during co-translational translocation activates RPL26 UFMylation

To determine the influence of protein translation on RPL26 UFMylation, we treated cells with drugs that block translation via distinct mechanisms. Intriguingly, anisomycin (ANS), which prevents peptidyl transfer and thus arrests ribosome in a specific conformation $^{39}$ during elongation strongly induced RPL26 UFMylation, and this phenotype was either completely abolished in 


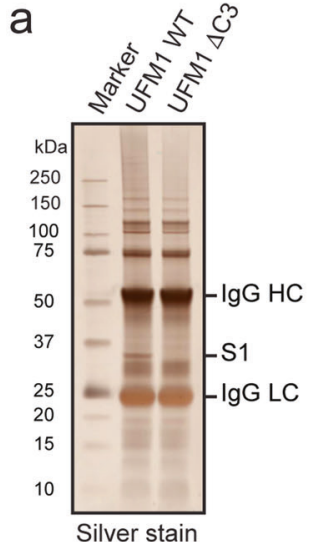

C
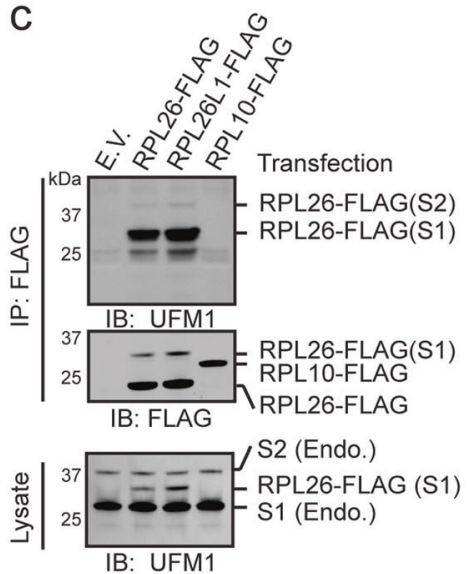

e

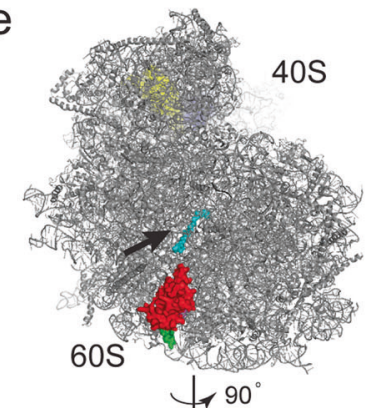

$f$

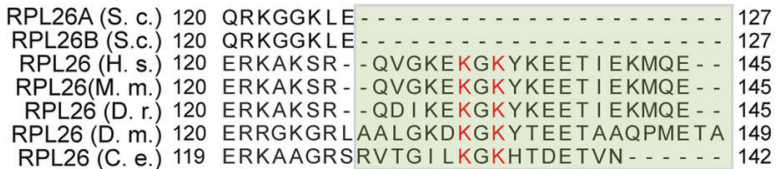

RPL26A (S. c.) 120 QRKGGKLE . . . . . . . . . . . . 127 RPL26 (H.s.) 120 ERKAKSR - QVVGKEKGKYKEETI EKMQE - 145 RPL26(M. m.) 120 ERKAKSR - - QVGKEKGKYKEET I EKMQE - - 145 RPL26 (D.m.) 120 ERRGKGRLAALGKDKGKYTEETAAQPMETA 149 RPL26 (C. e.) 119 ERKAAGRSRVTGI LKGKHTDETVN - . - . 142

9
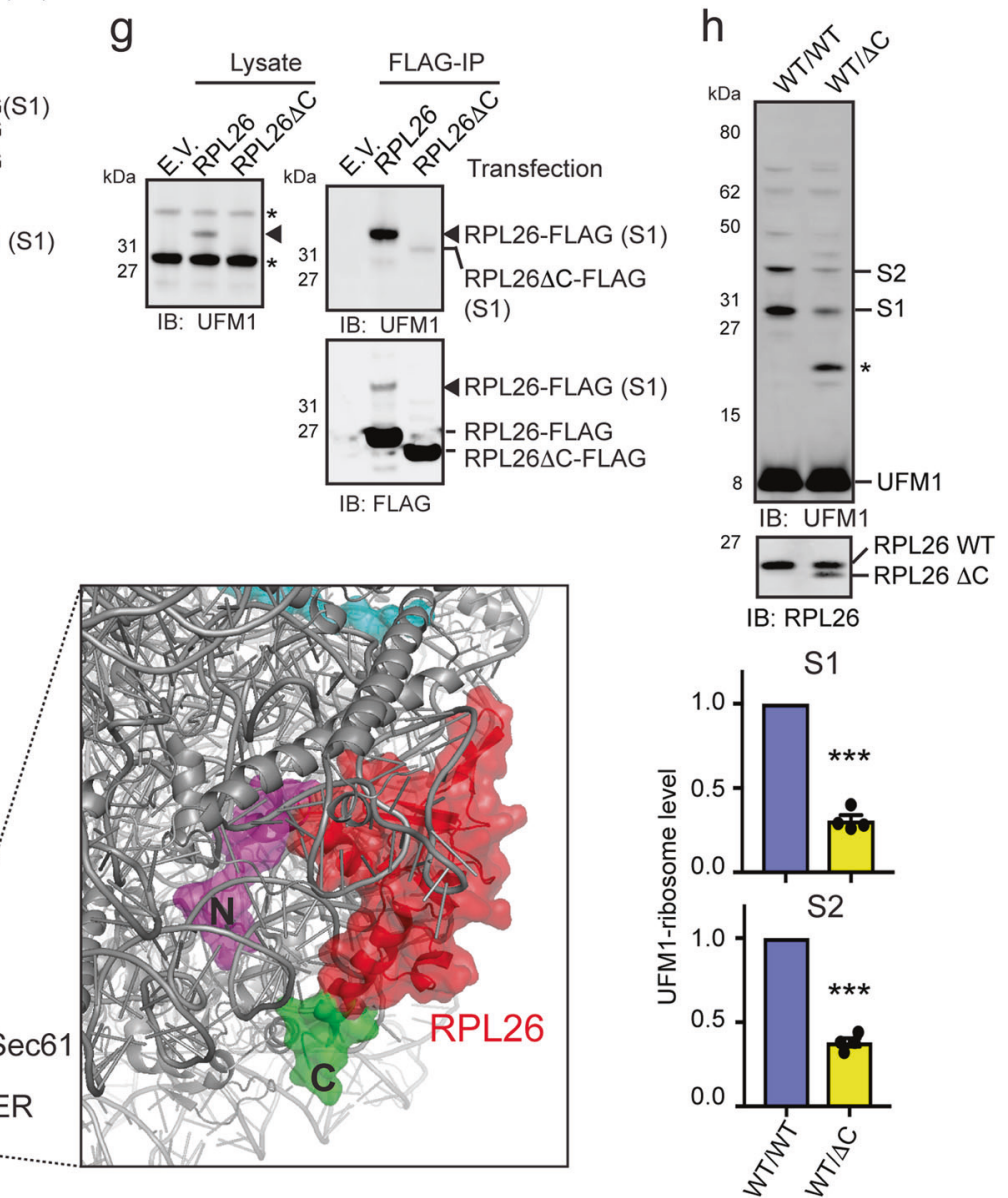

UFM1-deficient cells or diminished in cells lacking the RPL26 C-tail (Fig. 2a, Supplementary information, Fig. S5a-c). Polysome profiling showed that ribosome UFMylation in ANS-treated cells predominantly took place at $80 \mathrm{~S}$ (assembled) ribosomes with a small fraction on the dissociated 60S subunit (Fig. 2b). The early elongation inhibitor harringtonine ${ }^{40}$ and the elongation inhibitor cycloheximide moderately increased RPL26 UFMylation (Fig. 2c, d, Supplementary information, Fig. S5b, d), whereas puromycin, which released ribosome from mRNA ${ }^{41}$ had little effect (Fig. 2c, d, Supplementary information, Fig. S5e). Among a collection of stress-inducing compounds tested, arsenite and the ER stress inducer thapsigargin increased RPL26 UFMylation, but 
Fig. 1 RPL26 is UFMylated at two conserved lysine residues in its carboxyl tail. a Proteins purified following the scheme in Supplementary information, Fig. S2a were analyzed by SDS-PAGE and silver staining. HC heavy chain, LC light chain. $\mathbf{b}$ A scatter plot shows the peptide count for proteins identified by mass spectrometry from gel slices at the S1 position in a. c UFSP2 knockout HEK293T cells transfected with the indicated plasmids were lysed. Lysates were subjected to immunoprecipitation (IP) by FLAG antibodies under denaturing conditions. Precipitated proteins and a fraction of the lysates were analyzed by immunoblotting (IB). E.V. empty vector. Endo. endogenous S1, S2. d Immunoblotting of ribosome pellet $(P)$ and ribosome-free supernatant (S) fractions (see "MATERIALS AND METHODS" section) from the indicated cells. RPL26(C), antibodies recognizing C-terminus of RPL26, which might have lower affinity to modified RPL26 than the unmodified species, causing an underestimate of S1 and S2. Asterisks, non-specific bands. e Structural analysis of the UFMylation site on ribosome. The model is based on PDB 5LKS ${ }^{61}$ and 5AJ0.62 Red, RPL26; arrow, peptide exiting tunnel. The COOH- (contains Lys132 and Lys134) and NH2terminus of RPL26 are labeled in green and magenta, respectively. The small ribosomal subunits that undergo ubiquitination in cytosolic RQC are labeled in blue and yellow. f Sequence alignment of RPL26 COOH-tails. S.c. Saccharomyces cerevisiae, H.s. Homo sapiens, M.m. Mus musculus, D.r. Danio Rerio, D.m. Drosophila melanogaster, C.e. Caenorhabditis elegans. g RPL26-FLAG and RPL26 $\Delta$ C-FLAG immunoprecipitated (IP) from transfected UFSP2 knockout HEK293T cells by FLAG antibodies under denaturing conditions were analyzed by immunoblotting together with a fraction of cell lysates. The arrowheads indicate UFMylated RPL26-FLAG (S1). Asterisks, endogenous S1 and S2; E.V. empty vector. h Whole cell extracts from WT or RPL26 $\Delta$ C heterozygous (WT/ $\Delta$ C) K562 cells were analyzed by immunoblotting (asterisk, a protein that becomes UFMylated when endogenous target sites are missing). The graphs show quantification of S1 and S2 band intensity (error bars, SEM, $n=4$, ***P $P 0.001$, by paired two-tailed Student's $t$ test)

not to the extent observed with ANS (Fig. 2e, f). Because combining harringtonin or puromycin with ANS abrogated the effect of ANS (Fig. 2c, d), we conclude that RPL26 UFMylation is upregulated when ribosomes adopt a specific stalled conformation, which is induced by ANS treatment. This conclusion, together with the ER localization of the UFM1 ligase and UFMylated ribosomes (Supplementary information, Fig. S1f-h) prompted the idea that ribosome stalling during co-translational protein translocation might be a specific trigger for RPL26 UFMylation (Fig. 3a).

We therefore generated an ER-associated, model ribosome stalling substrate (ER_K20) consisting of an N-terminal signal sequence followed by a $\mathrm{N}$-glycosylation site, GFP, a stretch of Lys residues encoded by AAA codons and $\mathrm{COOH}$-terminal RFP (Fig. $3 \mathrm{~b}$ ). Poly-A sequence is known to induce ribosome stalling, ${ }^{8,42}$ impairing the synthesis of the RFP-bearing full-length protein. A construct without poly-A (ER_KO) served as a no-stalling control, whereas the corresponding $\mathrm{KO}$ and $\mathrm{K} 20$ constructs lacking the signal sequence served as cytosolic RQC controls (Cyto_KO and Cyto_K20).

Pulse labeling of nascent polypeptides with $\mathrm{S}^{35}$-methionine/ cysteine confirmed that the poly-A sequence induces ribosome stalling, generating partially translated arrested products (APs) for both ER_K20 and Cyto_K20 (Fig. 3c, lanes 3, 5 vs. 2, 4). Compared to Cyto-K20, translation arrest appeared more complete for ER_K20 as little read-through product was detected. Intriguingly, the ER_K20 construct generated two AP species migrating with Mr. of 50 and $53 \mathrm{kDa}$ (Fig. 3c, lane 5). The 53-kDa species was predominantly membrane-associated as demonstrated by fractionation (Fig. 3d, lane 2 vs. 1), and $N$-glycosylated given its sensitivity to Endo $\mathrm{H}$ digestion (Fig. 3c, lane 7 vs. 6). By contrast, the $50-\mathrm{kDa}$ species was more cytosolic and non-glycosylated (Fig. 3d). When we treated membranes from ER_K20-transfected cells with proteinase $\mathrm{K}$, the $53-\mathrm{kDa}$ species was largely protected by membranes whereas the $50-\mathrm{kDa}$ species was completely degraded (Fig. 3d, lanes 3-8). Thus, the 53-kDa protein (AP_ER) resides in the lumen while the $50-\mathrm{kDa}$ species (AP_Cyto) is cytosolically exposed. Ribosome pelleting combined with RNase A treatment (cleaved tRNA) demonstrated that $85 \pm 4 \%$ of the AP ER was co-sedimented with ribosomes (Fig. 3e, lane 5, Fig. 3f, lane 4 vs. 2). By contrast, full-length ER_KO was mostly present in the ribosome-free fraction (Fig. 3e, lane 4 vs. 3). Thus, even though AP_ER mostly resides in the lumen, its $\mathrm{C}$-terminus is still associated with ribosomes in part via the peptidyl-tRNA, consistent with the predicted topology of ER_K20 being stalled in the translocon during translocation. AP_Cyto by contrast is probably generated by ribosomes that have not yet engaged the translocon (Fig. 3e). This notion could be validated by co-IP, which showed that AP_ER, but not AP_Cyto was co-precipitated with the Sec61 translocon (Supplementary information, Fig. S6a). AP_Cyto was likely generated due to inefficient targeting of ER_K20 when the secretory pathway was saturated by over-expressed ER_K20 because reducing ER_K20 expression prevents the formation of AP_Cyto (see below).

Via immunoblotting we next analyzed RPL26 UFMylation in cells overexpressing these substrates. Expression of ER_K20, but not ER_K0 reproducibly increased S1 and S2 (Fig. 3g). As expected, RPL26 UFMylation was strictly dependent on translational stalling during co-translational translocation because Cyto_K20 did not increase UFMylated RPL26. We conclude that ribosome stalling during co-translational translocation at the ER is a specific trigger for ribosome UFMylation.

RPL26 UFMylation promotes degradation of translation-arrested ER proteins

Our findings raised the possibility that ribosome UFMylation may safeguard protein translocation at the ER to facilitate protein biogenesis. We therefore analyzed the stability of AP_ER in cells deficient in RPL26 UFMylation using a radiolabeling-based pulse chase assay. We initially used ER_K20-transfected cells because AP Cyto generated as a byproduct provided an ideal internal control. In WT cells, both AP_ER and AP_Cyto were short-lived, with $t_{1 / 2}$ of $\sim 40$ and $\sim 30 \mathrm{~min}$, respectively. By contrast, in cells lacking RPL26 UFMylation sites (RPL26 $\triangle$ C) or UFM1, AP_ER was significantly stabilized whereas the degradation of AP_Cyto proceeded normally (Fig. 4a-d). Re-expression of UFM1 in UFM1 CRISPR knockout cells restored AP_ER degradation (Supplementary information, Fig. S6b). These results suggested that RPL26 UFMylation facilitates the degradation of AP_ER but not that of AP_Cyto. By contrast, depletion of NEMF, a previously identified cytosolic RQC factor, did not significantly impact the turnover of overexpressed AP_ER. The efficacy of NEMF siRNA was confirmed by immunoblotting and by the observation that in the same experiment, it partially diminished AP_Cyto degradation (Supplementary information, Fig. S6c). Moreover, the degradation of Cyto_K20, a previously established cytosolic RQC substrate, ${ }^{8}$ was significantly inhibited by NEMF siRNA (Supplementary information, Fig. S6d). Likewise, treating cells with the proteasome inhibitor MG132 only selectively stabilized AP_Cyto but not AP_ER (Fig. 4e, lanes 9-12 vs. lanes 1-4; Fig. 4f). Degradation of ER_K20 was also not dependent on HRD1, an ERAD regulator essential for turnover of misfolded ER proteins bearing no transmembrane domains (Supplementary information, Fig. S6e, f). Altogether, these data suggest that AP_ER is eliminated by a RPL26 UFMylation-dependent mechanism that is distinct from cytosolic RQC or ERAD, while AP_Cyto is partially degraded by the previously established cytosolic RQC pathway.

To further confirm the role of UFMylation in degradation of translation-stalled ER proteins, we generated a GFP-bearing reporter that has no stop codon (ER_NS) (Supplementary 
a

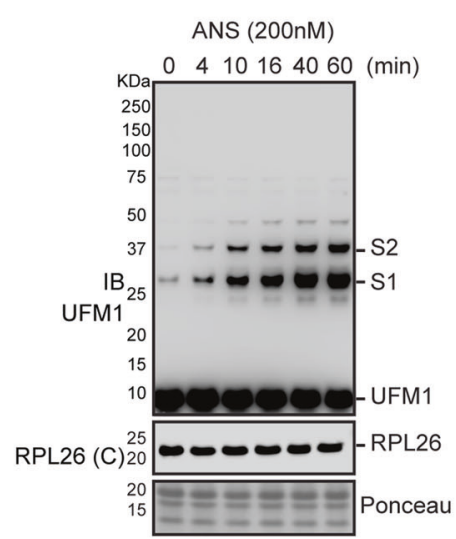

C
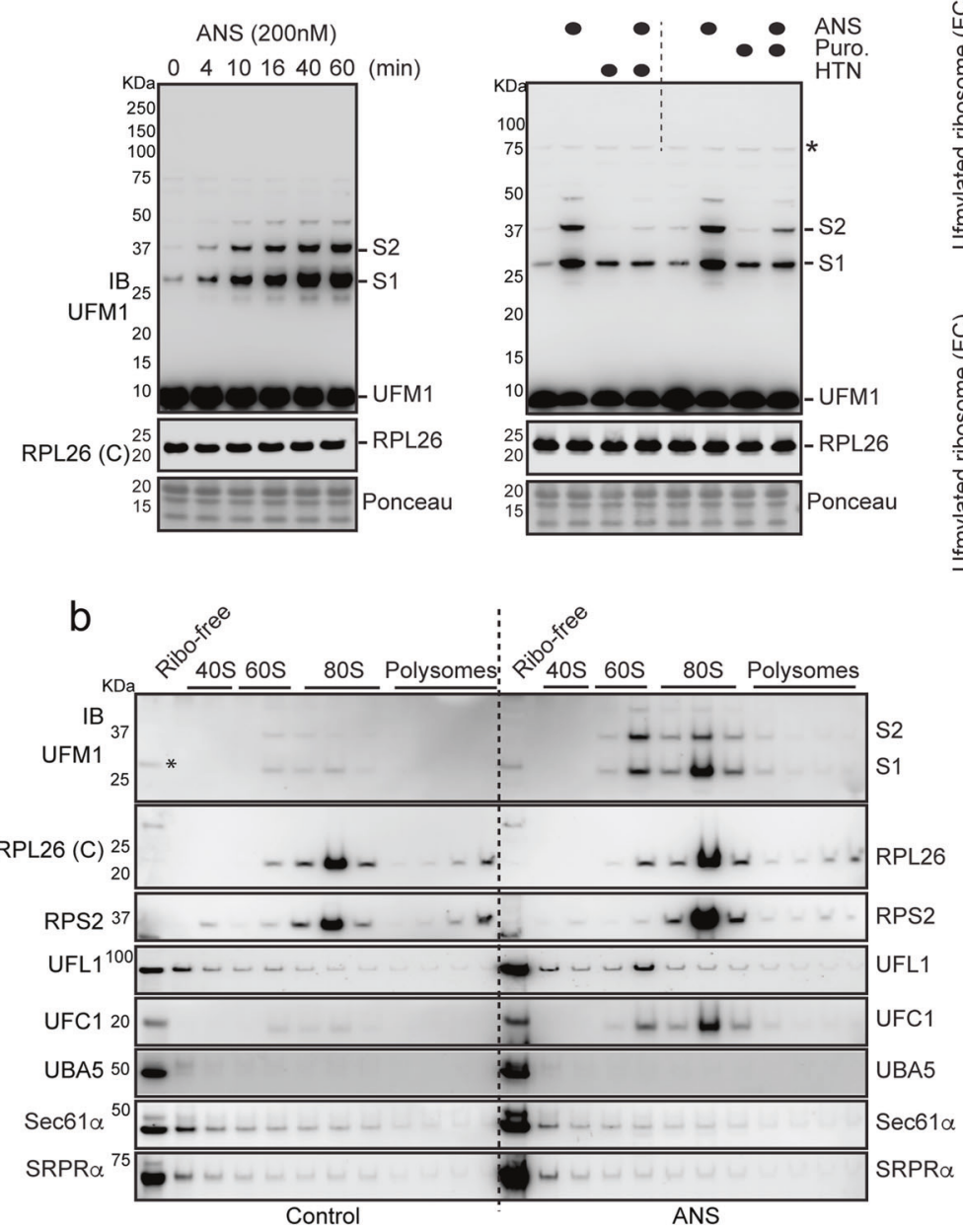

e

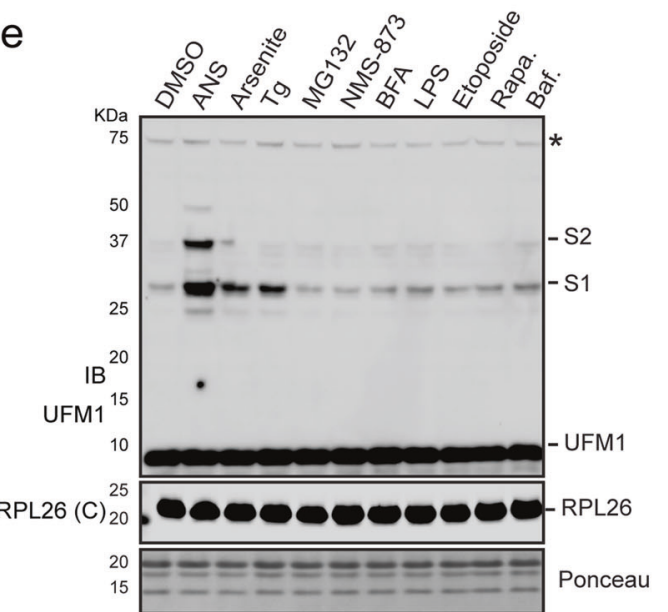

f d
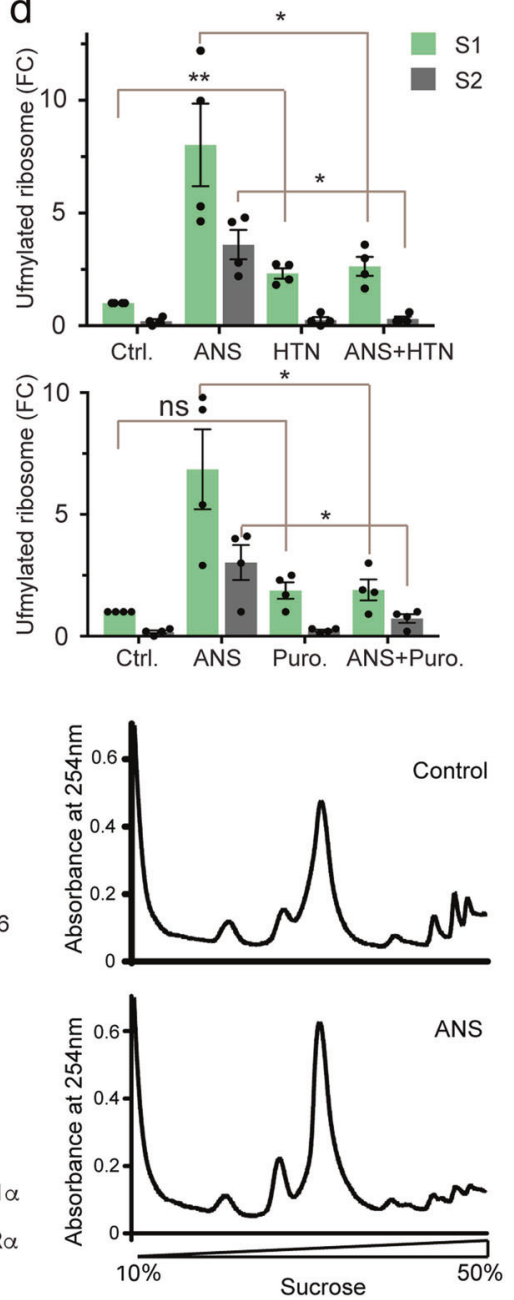

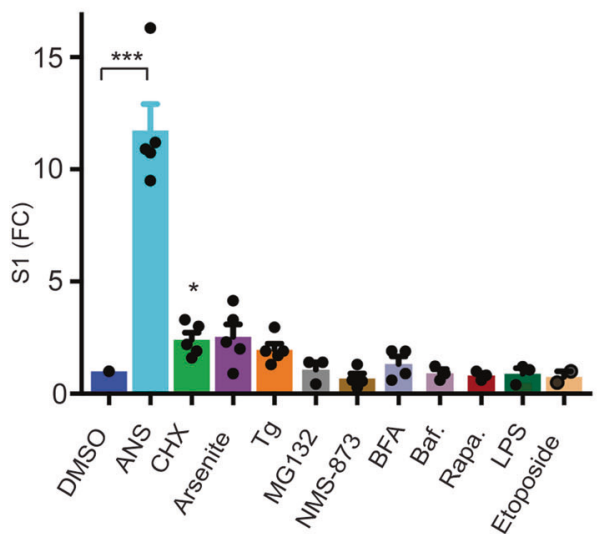

Fig. 2 Ribosome stalling during translation elongation induces RPL26 UFMylation. a Immunoblotting analysis of whole cell extracts from HEK293T cells treated with anisomycin (ANS) $(200 \mathrm{nM})$ for the indicated time periods. b Ribosomes from control or ANS-treated HEK293T cells were subjected to fractionation by continuous sucrose gradient. The collected fractions were analyzed by immunoblotting. The graphs show ribosome profiles revealed by absorbance $254 \mathrm{~nm}$ after ultracentrifugation. Asterisk, non-specific band. c Lysates from HEK293T cells treated for $1 \mathrm{~h}$ with ANS ( $200 \mathrm{nM})$, harringtonine (HTN) $(5 \mu \mathrm{M})$, or Puromycin (Puro.) $(10 \mu \mathrm{g} / \mathrm{mL})$ either individually or in combinations were analyzed by immunoblotting as in a. Asterisk, non-specific band. d Quantification of experiments in c (error bars, SEM, $n=4$, ns not significant, ${ }^{*} P<0.05$; ${ }^{*} P<0.01$ by two-tailed Student's $t$ test). Fold change (FC) is normalized by S1 level in control cells. e Immunoblotting analysis of whole cell extracts from HEK293T cells treated with the indicated compounds for $3 \mathrm{~h}$. Tg. Thapsigargin, Rapa. Rapamycin, Baf Bafilomycin A1, BFA Brefeldin A, LPS Lipopolysaccharides. $\mathbf{f}$ The graph shows the quantification of S1 level in e and in CHX-treated cells in Supplementary information, Fig. S5b. error bars, SEM, $n$ is indicated by the dots, ${ }^{*} P<0.05,{ }^{* * *} P<0.001$ by two-tailed Student's $t$ test 


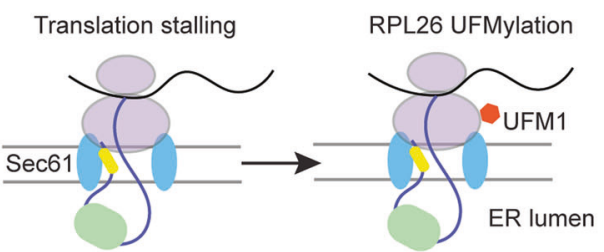

b

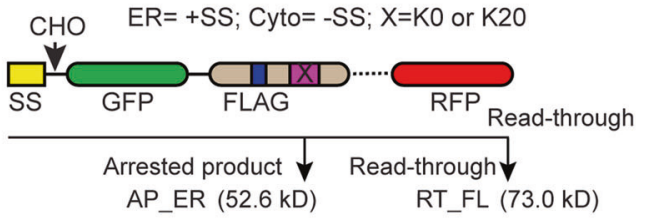

C

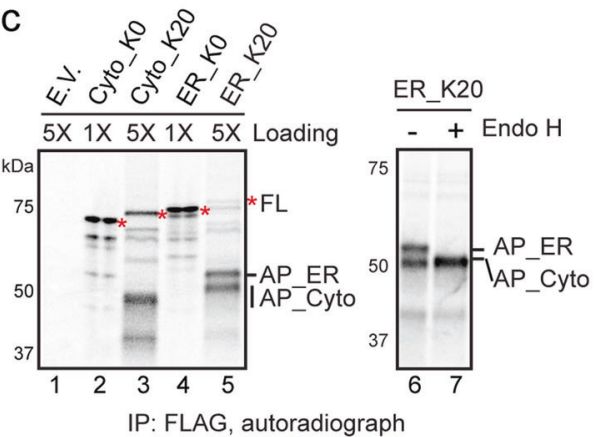

d

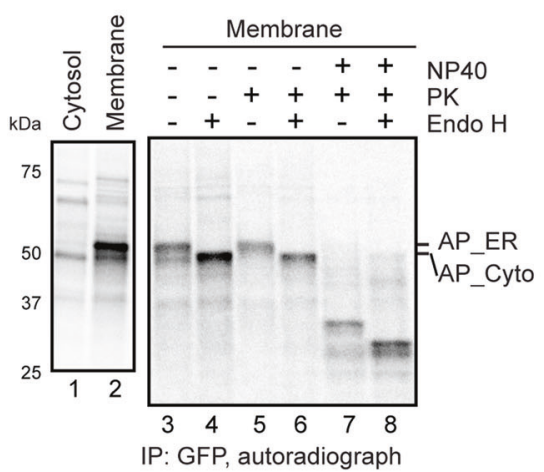

e

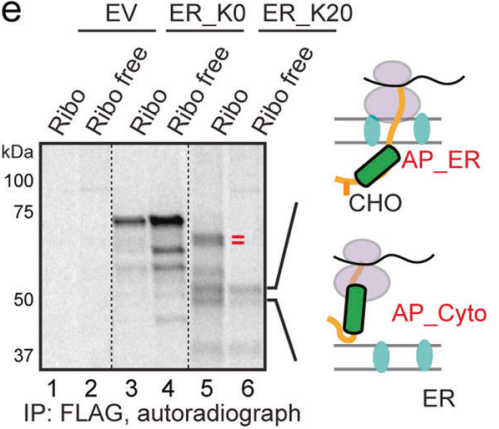

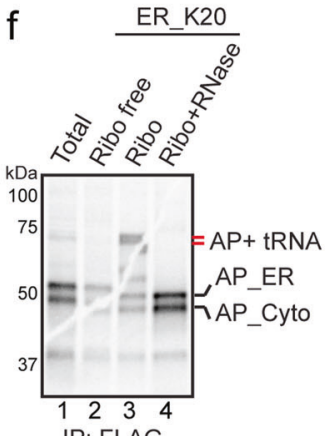

g

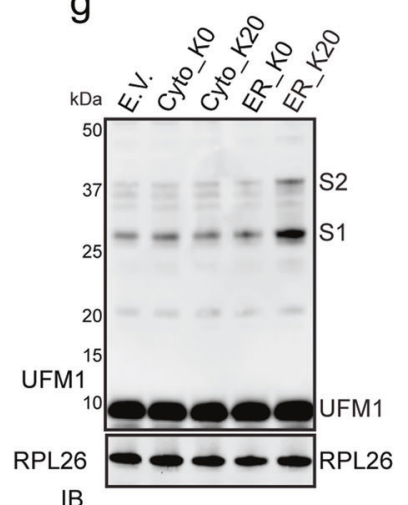

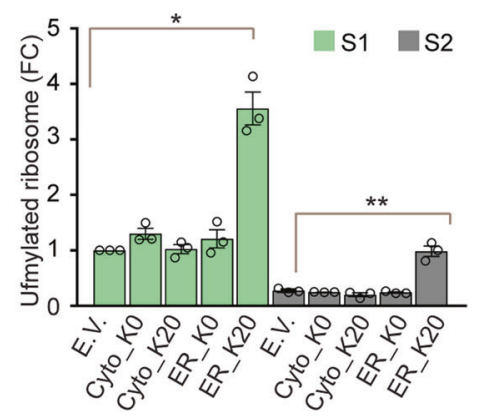

Fig. 3 Translation stalling during co-translational translocation at the ER is a specific trigger of RPL26 UFMylation. a A model of regulated ribosome UFMylation. b A schematic diagram showing the constructs used in $\mathbf{c}-\mathbf{g}$. SS signal sequence, CHO glycosylation site, $\mathrm{X}$ stalling sequence. c Radiolabeling analysis of nascent polypeptides in cells transfected with the indicated constructs. Asterisk, full-length (FL) proteins, AP arrested product. Sample in lane 5 was also treated with Endo H (lane 7) or untreated (lane 6). d Nascent AP_ER is protected by membranes. ER K20-transfected cells were radiolabeled, permeabilized, and fractionated into a cytosol and membrane fraction (lanes 1 and 2). Where indicated (lanes 5-8), the membrane fraction was treated with proteinase K (PK). Samples were subjected to immunoprecipitation with GFP antibodies to purify ER_K20. A fraction of the precipitated samples were Endo H-treated prior to SDS-PAGE (lanes 4, 6, and 8). e Ribosome purification by sucrose cushion shows that newly synthesized AP_ER is mostly co-sedimented with ribosomes (Ribo). Cells transfected with the indicated constructs were radiolabeled and lysed. The lysates were subjected to centrifugation through a sucrose cushion to isolate ribosome (Ribo) and ribosome-free fractions followed by immunoprecipitation with FLAG antibodies. $\mathbf{f}$ RNase treatment revealed a fraction of tRNA-linked AP_ER that is associated with ribosomes. Cells transfected with ER_K20 were radiolabeled and lysed. The resulting lysates were fractionated and processed as in e except that a fraction of ribosomes were treated with RNase before immunoprecipitation (lane 4). g ER_K20 overexpression induces ribosome UFMylation. Cells transfected as indicated were subjected to immunoblotting analysis. The graph shows the quantification of the experiments (error bars, sem, $n=3 ;{ }^{*} P<0.05$ and ${ }^{* *} P<0.01$ by paired, two-tailed Student's $t$ test). FC fold change

information, Fig. S6g). Previous studies have indicated that translation of mRNAs bearing non-stop codon is another major cause of ribosome stalling because ribosomes reaching the end of these mRNAs cannot be released. ${ }^{6,14,43}$ As expected, pulse chase experiments showed that ER_NS was a short-lived protein and its degradation was inhibited in cells treated with either sgRNA or siRNA that targets UFM1 (Supplementary information, Fig. S6g, h).

RPL26 UFMylation facilitates lysosomal transport of a translocation-arrested protein

To elucidate the degradation mechanism for translation-stalled nascent chains en route to the ER, we used chemical inhibitors to further characterize ER_K20 degradation. We first treated cells transfected with ER_K20 with the lysosomal inhibitor chloroquine (CQ). Now, in pulse chase experiments, AP_ER but not AP_Cyto was stabilized (Fig. 4e, lanes 5-8 vs. 1-4; Fig. 4g). Further, Brefeldin A, which blocks ER-to-Golgi trafficking, selectively stabilizes AP_ER (Fig. 4h, lanes $6-9$ vs. $2-5$, Fig. 4i). These data raise a possibility that AP_ER may be transported from the ER to lysosomes for degradation.

Next, we generated a HEK293T cell line (C20) that stably expressed ER_K20 6-fold lower than transient transfectants (Fig. 4h, lane 1 vs. 2). Now, all newly synthesized ER_K20 was targeted to the ER and glycosylated, offering a better tool to 
a

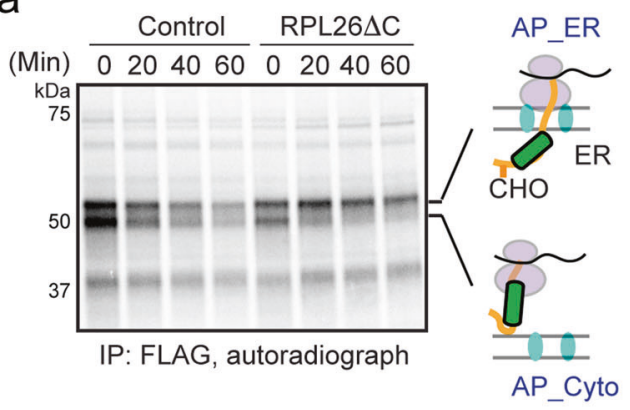

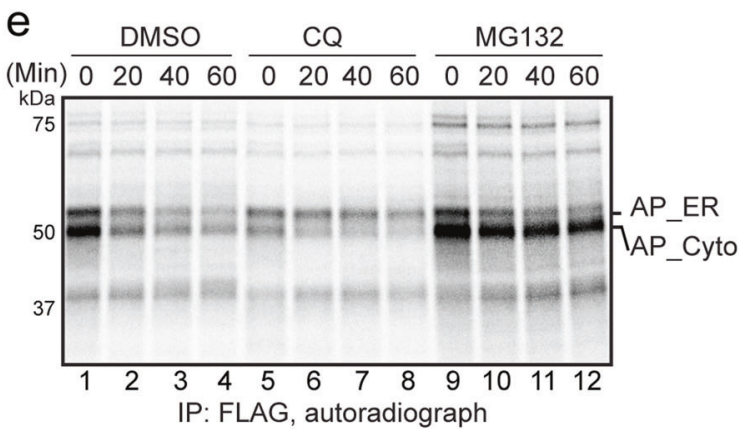

g

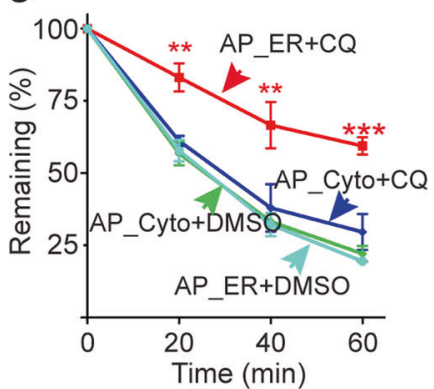

b

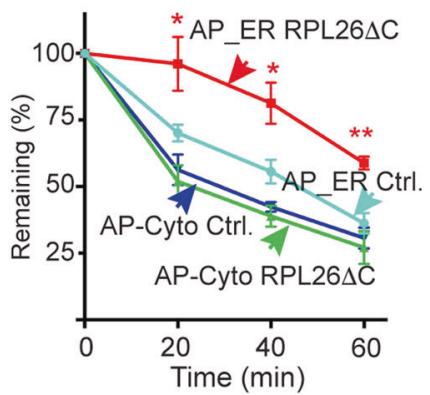

f

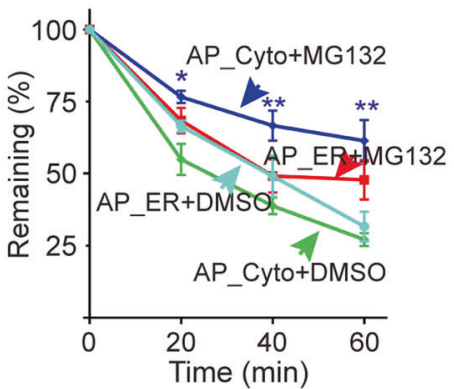

$\mathrm{h}$

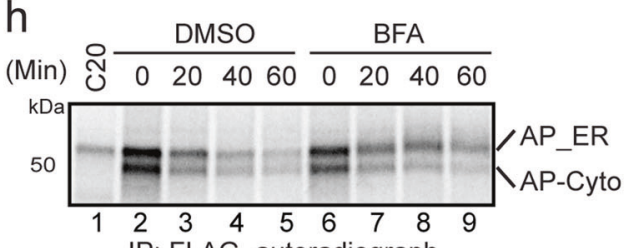

IP: FLAG, autoradiograph

i

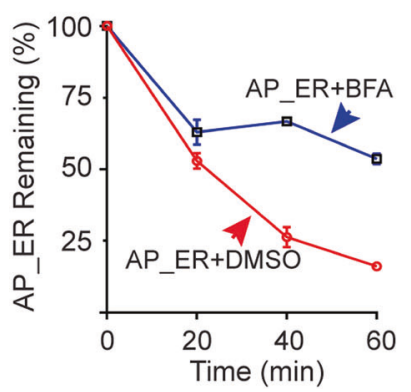

Fig. 4 RPL26 UFMylation promotes the turnover of a translation-arrested ER substrate in a lysosome-dependent manner. a Pulse-chase analysis of cells transfected with ER_K20 showed that RPL26 C-tail is required for efficient degradation of AP_ER, but not AP_Cyto. b Quantification of the experiments in a. c, d UFM1 is required for degradation of AP_ER. c As in a, except that control or UFM1 CRISPR knockout (KO) cells transfected with ER_K20 were used. d Quantification of the experiments in c. e The degradation of AP_ER and AP_Cyto was analyzed by pulse-chase using ER K20-transfected cells treated with DMSO (control), CQ (Chloroquine, $100 \mu M)$, or MG132 (20 $\mu M$ ). f, g Quantification of the experiments in e. Error bars represent SEM, $n=3$; ${ }^{*} P<0.05 ;{ }^{*} P<0.01 ;{ }^{* *} P<0.001$ by two-tailed Student's $t$ test in $(\mathbf{b}, \mathbf{d}, \mathbf{f}, \mathbf{g})$. h As in e, except that cells treated with DMSO and Brefeldin A (BFA, $10 \mu \mathrm{g} / \mathrm{mL}$ ) were analyzed (lanes 2-9) and that a sample from radiolabeled cells stably expressing ER_K20 (C20) was analyzed in lane 1. i Quantification of the experiments in h. Error bars represent data from two independent experiments

monitor AP_ER degradation. siRNA-mediated depletion of either UFM1 or UBA5 (E1 for UFM1) increased GFP intensity without affecting RFP, suggesting that UFMylation regulates specifically AP turnover without affecting translation read-through (Fig. 5a; Supplementary information, Fig. S7a). Additionally, fluorescence microscopy and fluorescence-activated cell sorting (FACS) analyses showed that while Brefeldin A and the lysosome inhibitor Bafilomycin A1 (Baf. A1) increased GFP intensity, MG132 only had a marginal effect (Fig. 5b, Supplementary information, Fig. S7b). These results further confirmed the degradation of AP_ER via a lysosome- and UFMylation-dependent pathway.

To test whether AP_ER itself is transported to lysosomes for proteolysis, we used live cell confocal fluorescence microscopy to examine the subcellular localization of AP_ER. In untreated C20 cells, AP_ER (green fluorescence) was present at low levels in a diffusive peri-nuclear pattern with vesicle-like puncta present in 
a

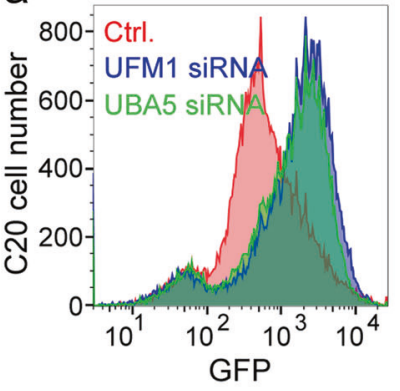

C
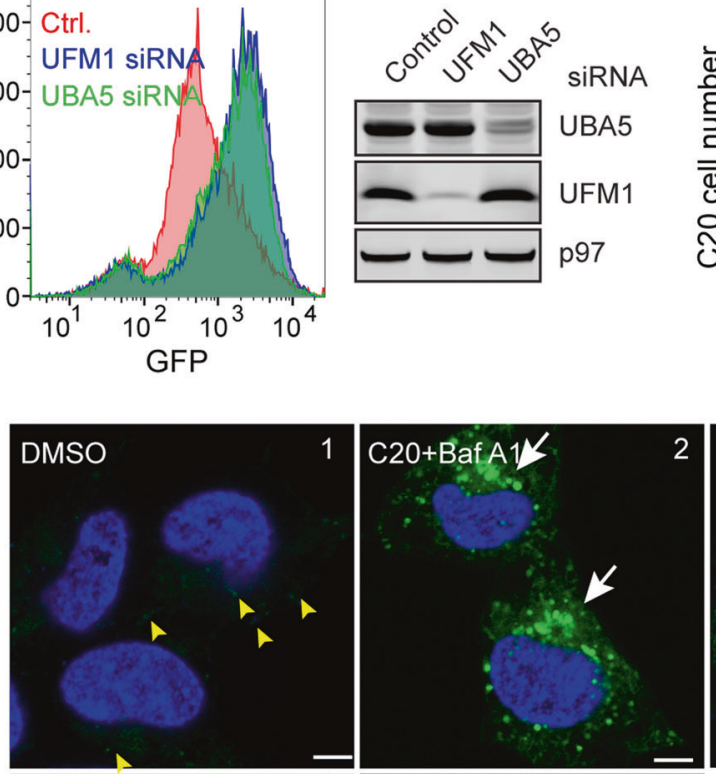

b

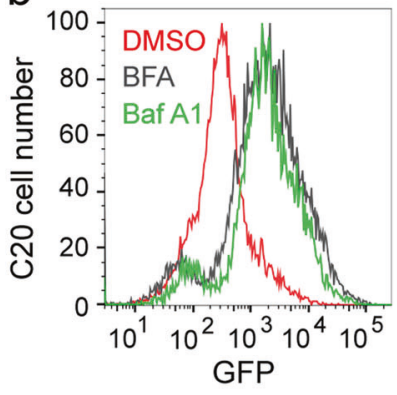

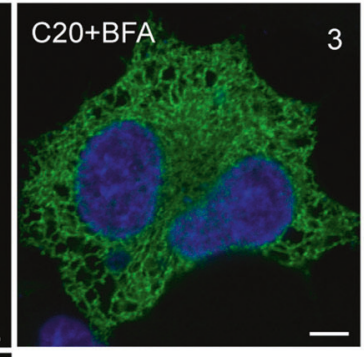

อ̊

6
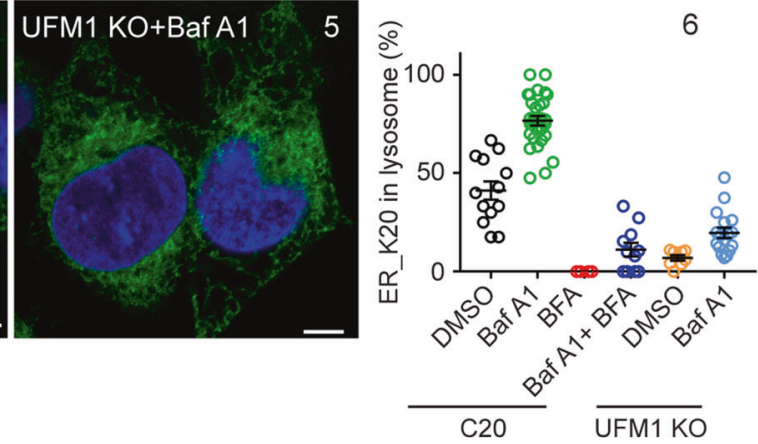

d
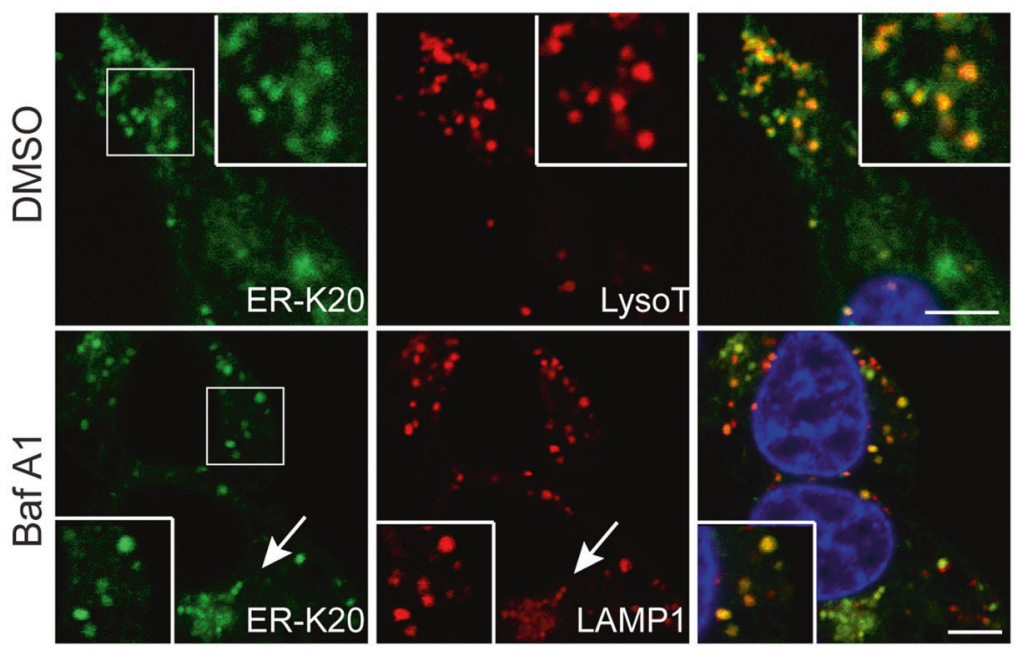

Fig. 5 RPL26 UFMylation promotes the targeting of translation-arrested ER substrate to lysosomes. a FACS analysis of GFP fluorescence in C20 cells that had been transfected with control (Ctrl.), UFM1, or UBA5 siRNA. A fraction of the cells were analyzed by immunoblotting to verify knockdown efficiency (right panels). b C20 cells treated with the indicated drugs were analyzed by FACS. c Confocal fluorescence microscopy analyses of ER_K20 localization in drug-treated C20 cells (panels 1-4) or Bafilomycin A1 (Baf. A1)-treated UFM1 null cells stably expressing ER_K20 (panel 5). Arrowheads in panel 1 indicate vesicle-localization of ER_K20. Arrows in panel 2 indicate ER_K20-bearing vesicles clustered in peri-nuclear regions. The graph in panel 6 shows the percentages of lysosome-localized cells in collected images (each contains 8-20 cells) from three independent experiments. d Co-localization of ER_K20 in C20 cells with the lysosome marker LysoTracker (LysoT, top panels) or mCherry-LAMP1 (bottom panels). The arrow indicates ER_K20 co-localized with LAMP1 in a peri-nuclear region. The insets show enlarged view of the indicated area. scale bars, $5 \mu \mathrm{m}$ 
$\sim 40 \%$ cells. Upon Baf. A1 treatment, AP_ER-associated green fluorescence was enhanced and concentrated in clustered perinuclear vesicles in $\sim 75 \%$ of cells (Fig. 5 c, panels $1,2,6$ ). Confocal imaging with dual fluorescence labels showed that these AP_ERcontaining vesicles were lysosomes because they could be labeled by either a LysoTracker dye or the lysosomal protein mCherryLAMP1 (Fig. 5d, Supplemental information, Movie S1). As expected, Brefeldin A-induced GFP signal was mostly ERlocalized (Fig. 5c, panels 3,6), even in cells co-treated with Baf. A1 (panels 4, 6). Because siRNA-dependent depletion of the ERphagy receptor FAM $134 \mathrm{~B}^{44}$ or the ribophagy receptor NUFIP1 ${ }^{45}$ did not affect AP_ER degradation (Supplementary information, Fig. S7c), ER_K20 appears to be targeted to lysosomes following the conventional Brefeldin A-sensitive ER exit pathway but not via an autophagy-related mechanism. Intriguingly, in UFM1 KO cells stably expressing ER_K20, AP_ER was mostly ER-localized regardless of Baf. A1 treatment (Fig. 5c, panels 5, 6), but re-expressing UFM1 in these cells rescued the lysosomal localization of ER_K20 after Baf. A1 treatment (Supplementary information, Fig. S7d, e). Likewise, in cells lacking the endogenous RPL26 UFMylation site (RPL26 $\triangle C$ ), Baf. A1 failed to induce ER K20 to accumulate in lysosomes (Supplementary information, Fig. S7d). Thus, arrested ER_K20 is primarily transported to lysosomes for degradation after exiting the ER, and this process is regulated by RPL26 UFMylation. Because immunostaining failed to detect RPL26 accumulation in lysosomes even in ANS-treated cells (Supplementary information, Fig. S7f), arrested ER_K20 must have been released from ribosomes prior to lysosomal targeting.

Ribosome UFMylation promotes ER protein biogenesis during erythroid differentiation

Elimination of translocation-stalled polypeptides is expected to safeguard ER protein biogenesis. Because mice lacking UBA5 or UFBP1 had severe defects in erythroid differentiation, ${ }^{24,25}$ we used in vitro erythroid differentiation models to dissect the physiological role of UFMylation-dependent protein quality control in ER protein biogenesis during cell differentiation. Treating K562 cells, a myelogenous leukemia cell line with hemin induced erythroid-like differentiation, as indicated by greatly increased expression of aand $\mathrm{y}$-globins, ${ }^{46}$ two prototypic erythrocyte proteins. Interestingly, coincident with hemoglobin induction, hemin treatment also increased RPL26 UFMylation (Fig. 6a). Ribosome UFMylation was also upregulated during erythropoietin (EPO)-induced erythroid differentiation in primary $\mathrm{CD}^{+} 4^{+}$hematopoietic stem and progenitor cells (HSPCs) (Supplementary information, Fig. S8a). These results corroborate the importance of ribosome UFMylation in erythroid differentiation.

Given that translocation-associated ribosome stalling is a primary trigger of ribosome UFMylation, the increased UFMylation phenotype hinted at more frequent translation stalling at the ER, which might arise from an increased demand for ER protein biogenesis. In agreement with this notion, when we purified ribosomes from hemin-treated and untreated K562 cells and performed RNA-seq to identify actively translated transcripts, we found that ribosomes from differentiated K562 cells were enriched for mRNAs encoding ER-targeted proteins (Fig. 6b, c, Supplementary information, Table S2). Likewise, our analysis of published RNA-seq data obtained from EPO-treated $\mathrm{CD}_{3} 4^{+} \mathrm{HSPCs}^{47}$ showed that during erythroid differentiation, the total transcripts encoding secretory/membrane proteins increased steadily until the polychromatic stage, while those for non-secretory proteins (excluding hemoglobin) were reduced (Supplementary information, Fig. S8b, c). Notably, hemin treatment also elevated the level of BiP protein (an ER resident heat shock protein required for ER protein biogenesis) and the stress-associated transcription factor ATF3 (Supplementary information, Fig. S8d, e, f). Since both BiP and ATF3 are unfolded protein response (UPR, also called ER stress) target genes, increased ER protein biogenesis might be inevitably associated with UPR activation under hemin-treated conditions. Intriguingly, UFM1 inactivation upregulated the basal level of BiP, but not ATF3, consistent with the reported functional interplay between UPR and UFMylation. ${ }^{38,48,49}$ However, under hemintreated condition, both UFM1-deficient and control cells had similar levels of BiP and ATF3 (Supplementary information, Fig. S8d, e, f). Thus, increased ER protein biogenesis during erythroid differentiation seems to cause more translocationassociated ribosome stalling, which in turn activates UPR and ribosome UFMylation. These observations provide an additional functional link between UFMylation-dependent protein quality control and ER protein biogenesis during erythroid differentiation.

If UFMylation-dependent protein quality control safeguards protein translocation to promote ER protein biogenesis, lack of UFM1 might cause a defect in protein secretion. Although our initial studies failed to detect a defect in basal protein secretion in UFM1 KO cells, we observed by qRT-PCR and immunoblotting that the expression of many proteins of the co-translational translocation pathway was consistently upregulated in cells defective in UFMylation (Supplementary information, Fig. S8g-j). This compensatory response presumably maintains sufficient number of functional translocons that meet the relatively low secretory demand under basal conditions. We therefore tested whether protein secretion under increased secretory load conditions (e.g., during erythroid differentiation) required UFM1.

We first tested endogenous Clusterin because this chaperone was dramatically induced in hemin-treated cells (Fig. 6b). Although UFM1 inactivation did not affect basal Clusterin secretion, hemin-amplified Clusterin secretion was significantly reduced in UFM1 KO K562 cells (Fig. 6d). Additionally, we tested the secretion of recombinant GFP bearing a signal sequence (ER_GFP) and found that its secretion was similarly diminished in hemin-treated UFM1 KO K562 cells (Supplementary information, Fig. S8k). Finally, consistent with the erythroid deficiency in mice lacking UFMylation, the production of hemoglobin $a$, a hallmark of erythroid differentiation, was significantly reduced in differentiating K562 cells bearing one allele of RPL26 that lacked the UFMylation site (Fig. 6e) or in UFM1-depleted CRISPR cells (Supplementary information, Fig. S8I). Collectively, these results underscore a critical role of UFMylation-dependent protein quality control in ER protein biogenesis, which supports erythroid differentiation.

\section{DISCUSSION}

Ribosome stalling on faulty mRNAs is known to take place frequently in eukaryotic cells. ${ }^{5,6}$ When occurring during protein translocation into the ER, translation arrests generate stalled polypeptides that could block the Sec61 translocon. It is unclear how cells resolve clogged translocons during co-translational ER targeting. Two reports showed that translation-arrested ER proteins could be ubiquitinated in a cell-free system and in yeast, hinting at a proteasomal degradation mechanism akin to either canonical ERAD or cytosolic RQC. ${ }^{17,50}$ However, this assumption has not been confirmed by experiments in mammalian cells. On a related note, in $S$. cerevisiae, translocon clogging can recruit an ER metalloprotease named Ste24 to cleave stalled polypeptides, but this mechanism is only applicable to a small number of proteins targeted post-translationally. ${ }^{51}$ Thus, the quality control mechanism safe-guarding co-translational ER translocation in mammalian cells has not been defined.

Our study together with a recent report ${ }^{35}$ identify RPL26 as a primary substrate of UFMylation. Study by Walczak and colleagues showed that cells defective in RPL26 UFMylation have undergone adaptive changes that elevate the expression of certain secretory proteins, but the precise function of RPL26 UFMylation has remained obscure. Our study additionally revealed a UFMylationdependent protein quality control mechanism, which turns over 
a

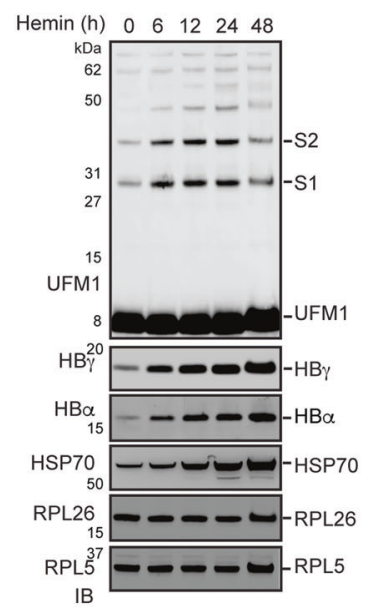

e

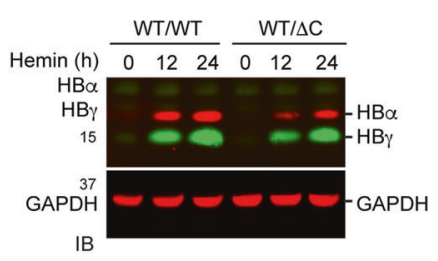

b

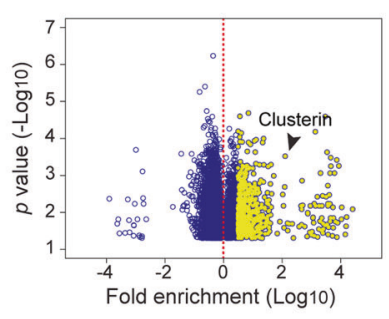

C

\begin{tabular}{lcl}
\hline GO Term & Number & $\mathrm{p}$-value \\
\hline Glycoprotein & 195 & $9.7 \mathrm{e}-4$ \\
Signal peptide & 151 & $3.2 \mathrm{e}-4$ \\
Plasma membrane & 184 & $4.3 \mathrm{e}-4$ \\
Membrane & 294 & $8.1 \mathrm{e}-3$ \\
\hline
\end{tabular}

d

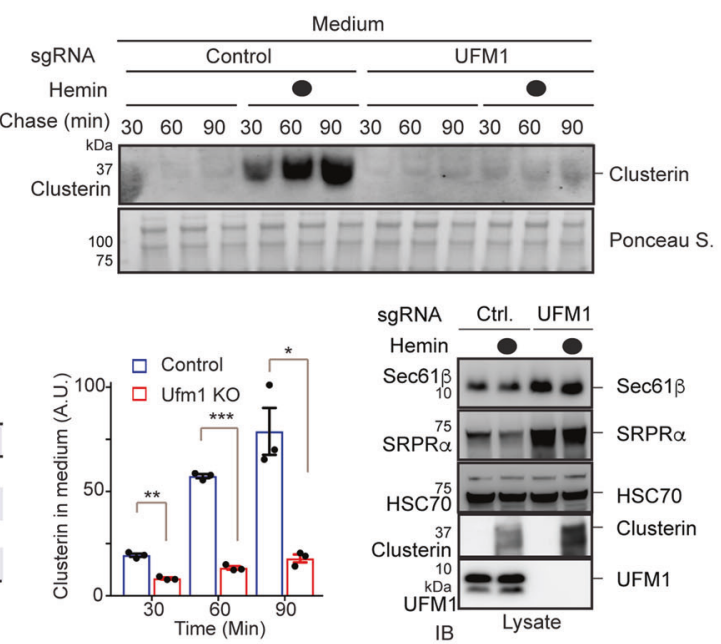

ig. 6 UFMylation promotes ER protein biogenesis in erythroid differentiation models. a Ribosome UFMylation is induced in hemin-treated K562 cells. b RNA-seq analysis of ribosome-associated mRNAs in hemin-treated vs. untreated K562 cells. Yellow circles indicate genes enriched by >threefold after hemin treatment. c Gene ontology analysis of the yellow-labeled transcripts in b shows an enrichment of membrane/ glycoproteins. d UFM1 KO K562 cells are defective in protein secretion during differentiation. Control or UFM1 KO K562 cells were treated with hemin $(50 \mu \mathrm{M})$ for $24 \mathrm{~h}$ or left untreated. Cells were incubated in fresh medium. Medium harvested at the indicated time points (top panels) or cell lysates prepared at the last time point (lower panels) were analyzed by immunoblotting (IB). The graph shows the quantification of Clusterin secretion. A.U. arbitrary units. Error bars, SEM, $n=3,{ }^{*} P<0.05, * * P<0.01,{ }^{* * *} P<0.001$ by two-tailed Student's $t$ test. e RPL26 C-tail is required for hemoglobin $\alpha$ production in differentiating K562 cells. Immunoblotting analysis of control (WT/WT) or K562 cells bearing one allele of RPL26 $\Delta \mathrm{C}(\mathrm{WT} / \Delta \mathrm{C})$ that were both treated with hemin as indicated. The graph shows the quantification of hemoglobin $\alpha$ (Hb $\alpha$ ) levels (error bars, SEM, $n=4,{ }^{*} P<0.01 ; * * * P<0.001$ by two-tailed Student's $t$ test). A.U. arbitrary units. f A model of ER-associated quality control of translocation-stalled proteins. Ribosome stalling during protein translocation induces RPL26 UFMylation, which facilitates the trafficking of stalled nascent chains to lysosomes for degradation

translation-arrested nascent polypeptides during protein insertion into the ER. Surprisingly, this mechanism resembles neither the canonical ERAD nor the well-established cytosolic RQC systems. Instead, ribosome stalling induces RPL26 UFMylation, which facilitates the trafficking of arrested products from the ER to lysosomes for degradation (Fig. 6f). This conclusion is supported by combined biochemical and imaging data, which reveal a defective trafficking and turnover pattern for a translation-arrested ER substrate in genetically edited cells defective in UFMylation or lacking the UFMylation sites on RPL26. By removing partially translated proteins, RPL26 UFMylation is expected to prevent the buildup of defective translation products in the ER, and also safeguard protein translocation to maintain secretory homeostasis. This notion is consistent with the elevated ER stress $^{32,38,48,49}$ and reduced protein secretion phenotypes observed in cells defective in UFMylation. The reduced protein secretion phenotype in hemin-treated UFM1 KO K562 cells is not a result of ER stress deregulation, because under hemin-treated conditions, control and UFM1-deficient cells activate UPR to a similar level. Additional studies are needed to elucidate how UFMylation deficiency impacts protein translocation at the ER.

Although proteasome inhibition or depletion of the cytosolic RQC factor NEMF does not significantly affect degradation of the tested translation-arrested AP_ER, conceptually, the trafficking of
AP_ER to lysosomes should involve ribosome recycling and the cleavage of the peptidyl-t-RNA linkage similarly as in cytosolic RQC. ${ }^{43,52,53}$ UFMylation is predominantly detected in $80 \mathrm{~S}$ ribosomes, suggesting that this modification occurs prior to ribosome splitting. Whether ribosome UFMylation serves as a signal to initiate or coordinate with these ribosome-associated RQC events remains to be elucidated, so are the factors involved in these steps. Additionally, since cells lacking UFM1 can still turn over AP_ER, just at a significantly reduced rate, it appears that a fraction of the stalled nascent chains could be degraded via a UFMylationindependent mechanism.

UFMylation-deficient mice are embryonic viable until $\sim 13$ days after gestation, ${ }^{25}$ suggesting that UFMylation is only essential during the development of specialized cell types. Consistently, RPL26 UFMylation is upregulated during erythroid differentiation, which correlates with an increase in ER protein biogenesis. However, since re-expression of UBA5 in erythroid cells in UBA5 knockout mice only rescue the erythroid differentiation defect without rescuing lethality, ${ }^{25}$ the role of ribosome UFMylation must not be limited to the erythroid lineages. We posit that cells during differentiation or under certain stress conditions may encounter more translocation-associated ribosome stalling due to increased demands in protein secretion or membrane protein biogenesis, and therefore would rely more on RPL26 UFMylation for survival. 
Our results suggest that improving translational fidelity or the efficiency of ER-associated degradation of translation-arrested proteins might be an effective strategy to rescue the developmental defects associated with UFMylation deficiency.

\section{MATERIALS AND METHODS}

Cell culture and transfection

The HEK293T, HEK293FT, K562 and HeLa cells were purchased from ATCC. HEK293T, HEK293FT and HeLa cells were maintained in Dulbecco's Modified Eagle Medium (DMEM, Corning) containing $10 \%$ fetal bovine serum (FBS) and antibiotics (penicillin/streptomycin, $10 \mathrm{U} / \mathrm{mL}$ ). $\mathrm{K} 562$ cells were cultured in Iscove's modified Dulbecco's medium (IMDM, ATCC) supplemented with 10\% FBS and antibiotics. All cell lines were maintained at $37^{\circ} \mathrm{C}$ in a $5 \% \mathrm{CO}_{2}$ humidified atmosphere. Hemin (Sigma) was dissolved at $1 \mathrm{mM}$ in $20 \mathrm{mM} \mathrm{NaOH}$ and filtered through a $0.22 \mu \mathrm{m}$ filter (Millipore). A final concentration of $50 \mu \mathrm{M}$ of hemin was used to induce erythroid differentiation of K562 cells as previously reported. ${ }^{54}$ Cell transfection was performed using TransIT-293 reagent (Mirus) for HEK293T or Lipofectamine 2000 (Invitrogen) for HeLa cells following the manufacturer's instructions. K562 cells were transfected by electroporation using Amaxa Cell Line Nucleofector Kit $\mathrm{V}$ in a nucleofector $2 \mathrm{~b}$ device (program T16, Lonza). For siRNAmediated gene silencing, Lipofectamine RNAiMAX (Invitrogen) was used according to the manufacturer's protocol.

Drug treatment

To test effects of translation inhibitors and other stress inducers on UFMylation, HEK293T cells were seeded at 0.5 million per well in a 12 -well plate. After $24 \mathrm{~h}$, medium was replaced with $1 \mathrm{~mL}$ fresh medium and DMSO (solvent) or different drugs were added at concentrations indicated in the figure legends. After treatment, cells were harvested, washed once with ice-cold PBS and directly boiled in Laemmli sample buffer at $95^{\circ} \mathrm{C}$ for $20 \mathrm{~min}$. Samples were analyzed by SDS-PAGE followed by immunoblotting.

Plasmids, chemicals, siRNAs and antibodies

Plasmids, chemicals, siRNAs and antibodies used in this study were listed in the Supplementary information, Table S3. Plasmids expressing mutant proteins were generated using QuikChange II Site-Directed Mutagenesis Kit (Agilent) following the manufacturer's instructions. Mutations were confirmed by Sanger sequencing. siRNAs were purchased from Invitrogen. The targeting sequences are: UFM1, 5'-CAGACUGCUGGAAAUGUUU-3'; UFL1, 5'GAGGAGUAAUUUUUACGGA-3'; and NEMF, 5'-GAUUAUGAGUACG UAAUUU-3'; UBA5, 5'-CUGCCUAAACAAGAGGUUA-3'.

To construct UFM1-expressing plasmids, UFM1 CDNA was PCRamplified from a human UFM1 CDNA clone (Origene) and inserted into pRK5 vector to generate both untagged or N-terminally HAtagged version. The pcDNA6-FLAG-SBP-UFM1 constructs were generated by inserting DNA fragment encoding FLAG-SBP (Streptavidin-Binding Peptide)-double tagged UFM1 between the BamHI and EcoRl sites in the pcDNA6-myc-His vector (Invitrogen). Lentiviral vectors expressing FLAG-UFM1 were generated by inserting a DNA fragment encoding FLAG-UFM1 into the FUGW vector that has a ubiquitin $C$ promoter. The lentiviral vector expressing ER_K20 was generated by inserting the open reading frame (ORF) encoding ER_K20 into the pLenti-Puro vector between the EcoRI and Agel sites. To generate pCMV6Entry-FLAG-RPL26 construct, DNA fragment encoding FLAG-RPL26 was PCR amplified and inserted between the Sgfl and Mlul sites in pCMV6- Entry vector (Origene). The pCMV6-Entry-RPL26 $\triangle C$-DDK construct was generated in the same way, except inserting a DNA fragment encoding the C-tail-truncated RPL26 (1-127 amino acids) followed by a Myc-FLAG tag.

The cytosolic ribosome stalling reporter constructs were a gift from Dr. Ramanujan Hegde from Addgene. To generate the
ER-targeted stalling constructs, oligonucleotides encoding $\mathrm{N}$-terminal signal sequence from MHC class I antigen (MVPCTLLLLLAAAL

APTQTRA) followed by a $N$-linked glycosylation site (Asn-X-Thr) (QDLPGNDNSTAGGS) were synthesized, annealed and inserted at the N-terminus of each cytosolic stalling construct. To remove the viral P2A (Porcine teschovirus-1 2A) motifs from the original stalling reporters, the following $P 2 A$-encoding sequences $\left(5^{\prime}-G G\right.$ CAGCGGCGCCACCAACTITCCCTGCTCAAGCAGGCCGGCGACGTGG AAGAGAATCCCGGCCCCGGTACT- $3^{\prime}$ and 5'-GCTACTAACTTCAGCCT GCTGAAGCAGGCTGGAGACGTGGAGGAGAACCCTGGACCT-3') were deleted by site-directed mutagenesis.

The ER-targeted non-stop construct (ER-NS) was generated based on the pEGFP-C1 plasmid (Clontech). The abovementioned $\mathrm{N}$-terminal signal sequence followed by the $N$-linked glycosylation site was inserted at the N-terminus of EGFP and all in-frame stop codons were mutated by site-directed mutagenesis.

The pX330-U6-Chimeric_BB-CBh-hSpCas9 vector was a gift from Dr. Feng Zhang from Addgene. The D10A mutation in Cas9 was introduced by site-directed mutagenesis to generate Cas9 D10A nickase. Two adjacent sgRNAs (single guide RNAs) were designed to target each gene according to the published protocol. ${ }^{55}$ Specificity of the designed sgRNA sequence was verified using Basic Local Alignment Search Tool (BLAST). The following sgRNA oligonucleotides were cloned into pX330-U6-Chimeric_BB-CBhhSpCas9 vectors:

UFM1_sgRNA_1: 5'-CTTTAAGATCACGCTGACGT-3'; UFM1_sgRNA_1: 5'-CTTCGACCTGAGGAAAAGAG-3'.

UFL1_sgRNA_1: 5'-CGGACGCCTGGGAAGAGATT-3'; UFL1_sgRNA_2: 5'-GGCCTGACTCGCAGTAGACG-3'.

UFSP2_sgRNA_1: 5'-AGTGACATAAACACCATTCC-3'; UFSP2 sgRNA_1: 5'-GCCATATATACACTGAACTG-3'.

RPL26_sgRNA_1: 5'-AGAAACCATTGAGAAGATGC-3'; RPL26_ sgRNA_1: 5'-TGCCCTTTTCCTTTCCTACT-3'.

Identification of RPL26 as a UFM1 substrate

Eight $15-\mathrm{cm}$ dishes of UFSP2 knockout HEK293T cells transiently transfected with FLAG-SBP-tagged WT UFM1 or UFM1 $\triangle \mathrm{C} 3$ mutant were harvested in $50 \mathrm{~mL}$ ice-cold PBS and washed once with PBS. Cells were then re-suspended in $7 \mathrm{~mL}$ hypotonic buffer $(20 \mathrm{mM}$ HEPES, pH 7.3, $10 \mathrm{mM} \mathrm{KCl}, 1 \mathrm{mM} \mathrm{MgCl}, 1 \mathrm{mM}$ DTT, $2 \mathrm{mM} \mathrm{N}$ Ethylmaleimide, plus a Roche EDTA-free Protease Inhibitor Cocktail) and incubated on ice for $5 \mathrm{~min}$. Cells were then homogenized on ice using a Dounce homogenizer, and sucrose was added to a final concentration of $250 \mathrm{mM}$. Cells were centrifuged at $1,000 \times g$ for $5 \mathrm{~min}$, and the resulting post-nuclear supernatant (PNS) was centrifuged at $4^{\circ} \mathrm{C}$ at $100,000 \times g$ for $20 \mathrm{~min}$ in a TLA 100.4 rotor. The membrane pellet was washed once with ultrapure water and solubilized in $6 \mathrm{~mL}$ NP40 lysis buffer $(20 \mathrm{mM}$ Tris, pH 7.4, $150 \mathrm{mM}$ $\mathrm{NaCl}, 1 \mathrm{mM}$ EDTA, $2 \mathrm{mM} \mathrm{MgCl} 2,0.5 \% \mathrm{NP} 40$ with a protease inhibitor cocktail) at $4{ }^{\circ} \mathrm{C}$ for $25 \mathrm{~min}$, followed by centrifugation at $17,000 \times g$ for $10 \mathrm{~min}$. Cleared membrane extracts were first incubated with $300 \mu \mathrm{L}$ prewashed slurry of anti-FLAG M2 beads (Sigma) for $90 \mathrm{~min}$ at $4{ }^{\circ} \mathrm{C}$ with constant rotation to remove free FLAG-SBP-UFM1 because pilot purification study showed that UFM1-RPL26 conjugates did not bind to FLAG antibody under this native purification condition. The lysate was then collected by passing through a Bio-rad purification column. SDS and DTT were added to cleared lysates at a final concentration of $1 \%$ and $5 \mathrm{mM}$, respectively. The lysate was then boiled at $95^{\circ} \mathrm{C}$ for $12 \mathrm{~min}$, and then subjected to centrifugation at $17,000 \times g$ for $10 \mathrm{~min}$ to pellet any insoluble materials. The cleared supernatant was diluted 5fold with NP40 lysis buffer and incubated with $400 \mu \mathrm{L}$ prewashed slurry of anti-FLAG M2 beads for $2.5 \mathrm{~h}$ at $4{ }^{\circ} \mathrm{C}$ using a head-over-tail rotator. The beads were then washed extensively with NP40 wash buffer (20 mM Tris, pH 7.4, $150 \mathrm{mM} \mathrm{NaCl}, 1$ mM EDTA, 2 mM MgCl${ }_{2}$, $0.05 \%$ NP40) at $4{ }^{\circ} \mathrm{C}$. Bound proteins were eluted by boiling the beads in $60 \mu \mathrm{L}$ Laemmli sample buffer at $95^{\circ} \mathrm{C}$ for $10 \mathrm{~min}$. A small 
fraction of the eluate was analyzed using SDS-PAGE followed by silver-staining following the standard protocol (SilverQuest, Life Technologies). For Mass spectrometry analysis, the remaining eluate was resolved by SDS-PAGE followed by Colloidal Coommassie blue staining (Thermo Fisher Scientific). The desired protein band were excised and analyzed by the Taplin Mass Spectrometry Facility at Harvard Medical School.

In vitro reconstitution of ribosome UFMylation

In vitro ribosome UFMylation was performed using semipermeabilized UFM1 knockout HEK293T cells. Briefly, 10 million cells were pre-treated with $200 \mathrm{nM}$ anisomycin for $20 \mathrm{~min}$. After washing with PBS, cells were resuspended in $320 \mu \mathrm{L}$ PB buffer (25 mM HEPES, pH 7.3, $115 \mathrm{mM} \mathrm{KOAc,} 2.5 \mathrm{mM} \mathrm{MgCl}_{2}, 5 \mathrm{mM} \mathrm{NaOAc}$ and $0.5 \mathrm{mM}$ EGTA) containing $0.055 \%$ digitonin, $1 \mathrm{mM}$ DTT and complete protease inhibitors, and were incubated on ice for $5 \mathrm{~min}$, followed by centrifugation at $14,000 \times g$ for $10 \mathrm{~min}$ at $4{ }^{\circ} \mathrm{C}$. After washing the membrane pellet with $\mathrm{PB}$ buffer, the membranes were resuspended in $300 \mu \mathrm{L}$ PB buffer and divided into three equal portions. To reconstitute ribosome UFMylation, a complete reaction mixture containing purified UFM1 $(2.5 \mu \mathrm{g} / \mu \mathrm{L})$, UBA5 $(0.25$ $\mu \mathrm{g} / \mu \mathrm{L}), \mathrm{UFC} 1(\mathrm{E} 2,0.6 \mu \mathrm{g} / \mu \mathrm{L})$ and ATP were added to one portion. The other two portions received a reaction mixture lacking either ATP or UFM1. The cells were incubated at $37^{\circ} \mathrm{C}$ for $30 \mathrm{~min}$ and reaction was stopped by addition of $4 \times$ Laemmli sample buffer and boiling at $95^{\circ} \mathrm{C}$ for $10 \mathrm{~min}$. Samples were analyzed by SDSPAGE followed by immunoblotting using both anti-UFM1 and anti-RPL26 (sigma, R0870) antibodies.

\section{Lentivirus production and transduction}

K562 cell line stably expressing FLAG-UFM1 and HEK293T cells stabling expressing ER_K20 were generated by lentiviral transduction. For Lentivirus production, two $15-\mathrm{cm}$ dishes of HEK293FT cells were seeded at $40 \%$ confluence. In the next day, $1 \mathrm{~h}$ prior to transfection, medium was replaced with $13 \mathrm{~mL}$ prewarmed Opti-MEM medium (Life Technologies). Transfection was performed using Lipofectamine 2000 and PLUS reagent (Life Technologies). For each dish, $6.8 \mu \mathrm{g}$ pCMV-VSV-G, $10.1 \mu \mathrm{g}$ psPAX2 (Addgene), $13 \mu \mathrm{g}$ gene-specific lentiviral plasmids and $135 \mu \mathrm{L}$ PLUS reagent (Invitrogen) were added to $4 \mathrm{~mL}$ Opti-MEM as mixture $A$, which is then mixed with mixture $B$ containing $68 \mu \mathrm{L}$ lipofectamine 2000 and $4 \mathrm{~mL}$ Opti-MEM. The complete mixture was incubated for $20 \mathrm{~min}$ at RT before being added to cells. After $6 \mathrm{~h}$, the medium was changed to $25 \mathrm{~mL}$ D10 medium (DMEM medium with $10 \%$ FBS and $1 \%$ Bovine Serum Albumin) with antibiotics (penicillin/streptomycin, $10 \mathrm{U} / \mathrm{mL}$ ) for virus production. After $60 \mathrm{~h}$ of incubation, virus-containing medium from two culture dishes were combined and centrifuged at 3,000 rpm at $4{ }^{\circ} \mathrm{C}$ for $10 \mathrm{~min}$ to pellet cell debris. The supernatant was filtered through a $0.45 \mu \mathrm{m}$ low protein-binding membrane (Steriflip HV/PVDF, Millipore). To concentrate lentivirus, the cleared supernatant was ultracentrifuged at $24,000 \mathrm{rpm}$ for $2 \mathrm{~h}$ at $4{ }^{\circ} \mathrm{C}$ using the JA25.50 rotor (Beckman). Virus was resuspended overnight in 180 $\mu \mathrm{L}$ D10 medium at $4{ }^{\circ} \mathrm{C}$. Virus was aliquoted, flash-frozen in liquid nitrogen and stored at $-80^{\circ} \mathrm{C}$.

For transduction, cells were seeded in 12-well plates and transduced via spin infection. Briefly, one million cells were resuspended with standard medium supplemented with $8 \mu \mathrm{g} / \mathrm{mL}$ polybrene (Sigma) and titrated virus amount, then seeded into one well of a 12-well plate. The plate was centrifuged at $1,000 \times g$ for $2 \mathrm{~h}$ at $37^{\circ} \mathrm{C}$ and further incubated for $1 \mathrm{~h}$ in the incubator before the medium was replaced with fresh medium. After $48 \mathrm{~h}, \mathrm{~K} 562$ cells expressing FLAG-UFM1 were validated by immunostaining and subsequently analyzed by immunofluorescence microscopy. For cells expressing ER_K20 (C20), HEK293T cells bearing control sgRNA (C20) or UFM1 specific sgRNA were cultured in the presence of puromycin for 7 days and then sorted by green fluorescence using the fluorescence-activated cell sorting (FACS).
Immunoprecipitation under denaturing and native conditions To detect UFMylation in cells, HEK293T cells grown in a 6-well plate were harvested and washed once with ice-cold PBS. After brief centrifugation at $10,000 \times g$ for $15 \mathrm{~s}$, cells were resuspended in $100 \mu \mathrm{L}$ ice-cold PBS and mixed with $100 \mu \mathrm{L}$ of $2 \%$ SDS, $5 \mathrm{mM}$ $\mathrm{DTT}$, followed by immediate boiling at $95^{\circ} \mathrm{C}$ for $10 \mathrm{~min}$ to disrupt protein complexes. Cell extracts were then diluted 5 -fold by NP40 lysis buffer ( $20 \mathrm{mM}$ Tris, pH 7.4, $150 \mathrm{mM} \mathrm{NaCl}, 1 \mathrm{mM}$ EDTA, $2 \mathrm{mM}$ $\mathrm{MgCl}_{2}, 0.5 \% \mathrm{NP} 40$ ) containing $2 \mathrm{mM} \mathrm{N}$-Ethylmaleimide (NEM) (Sigma) and EDTA-free Protease Inhibitor Cocktail (Roche) and rocked for $30 \mathrm{~min}$ at $4{ }^{\circ} \mathrm{C}$. After centrifugation at $17,000 \times \mathrm{g}$ for $10 \mathrm{~min}, 600 \mu \mathrm{L}$ supernatant was recovered and incubated with $40 \mu \mathrm{L}$ pre-equilibrated anti-FLAG M2 beads at $4^{\circ} \mathrm{C}$ for $1 \mathrm{~h}$ using a head-over-tail rotator. Beads were then washed twice with NET buffer (Tris- $\mathrm{HCl}, \mathrm{pH} 7.4,50 \mathrm{mM}, \mathrm{NaCl} 150 \mathrm{mM}, \mathrm{NP}-40 \quad 0.5 \%$ ) containing $0.1 \%$ SDS. Beads were then incubated with $50 \mu \mathrm{L}$ Laemmli sample buffer and heated at $95^{\circ} \mathrm{C}$ for $10 \mathrm{~min}$ before SDSPAGE and immunoblotting analyses.

To detect the interaction of AP_ER with the translocon, HEK293T cells were seeded in a $10 \mathrm{~cm}$ dish and grown for $24 \mathrm{~h}$ followed by transfection of $5 \mu \mathrm{g}$ ER_K20-expressing plasmid. After $24 \mathrm{~h}$, cells were starved in $6 \mathrm{~mL}$ starvation medium (DMEM medium free of methionine and cysteine) for $30 \mathrm{~min}$, labeled in $1 \mathrm{~mL}$ starvation medium supplemented with $10 \mathrm{mCi}\left[{ }^{35} \mathrm{~S}\right]$-methionine/cysteine for $20 \mathrm{~min}$. Cells were then lysed in $1.3 \mathrm{~mL}$ Digitonin lysis buffer (25 mM HEPES, pH 7.3, $250 \mathrm{mM}$ KOAc, $10 \mathrm{mM} \mathrm{MgCl}{ }_{2}$, $5 \mathrm{mM} \mathrm{NaOAc}$ and $0.5 \mathrm{mM}$ EGTA) containing $2 \%$ digitonin, $1 \mathrm{mM}$ DTT and complete protease inhibitors on ice for $20 \mathrm{~min}$. Sucrose was added to a final concentration of $250 \mathrm{mM}$. The sample was centrifuged at $20,000 \times g$ for $5 \mathrm{~min}$ at $4{ }^{\circ} \mathrm{C}$. Cleared cell lysate was then incubated with $10 \mu \mathrm{L}$ pre-immune or Sec61 $\beta$ serum for $1 \mathrm{~h}$ at $4{ }^{\circ} \mathrm{C}$. Protein A beads were used to precipitate antibody protein complexes. Precipitated materials were washed twice with digitonin wash buffer (25 mM HEPES, pH 7.3, $115 \mathrm{mM} \mathrm{KOAc,}$ $10 \mathrm{mM}$ Mg KOAc, $5 \mathrm{mM} \mathrm{NaOAc}, 0.2 \%$ digitonin, and $0.5 \mathrm{mM}$ EGTA) and bound proteins were eluted by boiling in $60 \mu \mathrm{L}$ SD buffer (1\% SDS, $5 \mathrm{mM} \mathrm{DTT).} \mathrm{One-third} \mathrm{of} \mathrm{the} \mathrm{samples} \mathrm{were} \mathrm{analyzed}$ directly by SDS-PAGE whereas the remaining samples were diluted by 9 -fold NP40 lysis buffer, followed by immunoprecipitation with FLAG antibodies.

Generation of RPL26 $\Delta$ C knock-in cell line by CRISPR/Cas9mediated homology-directed DNA repair (HDR)

CRISPR/Cas9-mediated HDR was used to endogenously edit RPL26 genomic locus (encoding amino acids 128-145) with a DNA sequence encoding the GFPs11 tag (GGGSRDHMVLHEYVNAAGIT, corresponding to the 11 th $\beta$-strand of the superfolder GFP). ${ }^{56}$ The GFP11-coding sequence also contains a BamHI cleavage site. For HEK293T cells, cells were seeded at 0.5 million per well in a 6-well plate. After $24 \mathrm{~h}$, cells were co-transfected with pX330-Cas9(D10A) vectors expressing two individual sgRNAs ( $2 \mu \mathrm{g}$ each) targeting a site in proximity to the RPL26 C-terminal sequence together with $100 \mu \mathrm{g}$ single-stranded HDR template (Ultramer, Integrated DNA Technologies) as following: 5'-AGGTGGTTATCACTAGGCTAAAAC TGGACAAAGACCGCAAAAAGATCCTCGAACGGAAAGCCAAATCTCG ACAGGGTGGTGGATCCCGGGACCACATGGTGCTGCACGAGTACGT GAACGCCGCCGGCATCACATAAAGTAATCTTATATACAAGCTITGA TTAAAACTTGAAACAAAGAGCCTGTGTITGTGTGTGGT-3'.

In the HDR template, the sgRNA-targeting sites were synonymously mutated leaving amino acid sequence unaffected. For K562 cells, 1 million cells were electroporated with pX330 Cas9 (D10A)-sgRNA-expressing vectors together with the HDR template. Cells were incubated for $48 \mathrm{~h}$ before being transfected with pCMVmGFP1-10. Cells were then expanded for $48 \mathrm{~h}$ prior to FACS based on reconstituted GFP fluorescence. GFP-positive cells were either single-cell sorted into 96-well plates or collected as a pooled population. GFP-negative cells were also sorted and pooled as control. Genomic DNA was extracted using the Wizard Genomic 
DNA Purification Kit (Promega) and used for PCR validation of successful gene editing. The following primers were used to amplify the genomic region around the insertion site: Forward: $5^{\prime}-$ CATCCACCTACCCCAGGTTG-3' and Reverse: 5'-ACACCCCTCCAGC TTCTGATA-3'. PCR products were purified, equally divided into two aliquots. One aliquot was subjected to BamHI digestion, and the other is untreated. The resulting PCR fragments were resolved in a $1.5 \%$ agarose gel, stained with SYBR Safe DNA Stain (Invitrogen) and imaged using Fuji LAS-4000. For K562 cells, all validated heterogeneous knock-in clones were pooled for further experiments.

Live cell imaging

Fifty thousand cells were seeded in fibronectin-coated chamber in a $\mu$-slide 8-well chamber coverslip (ibidi) and incubated overnight before drug treatment. Prior to imaging, indicated drugs were added to the cell and incubated for $3 \mathrm{~h}$. $30 \mathrm{~min}$ before imaging, LysoTracker $^{\text {TM }}$ red DND-99, Hoechst 33342 (Invitrogen), or both were added to the medium to stain lysosomes and nucleic acids, respectively. Immediately before imaging, the medium was replaced by fresh imaging medium containing respective drugs. Live cell confocal images were acquired using the LSM 780 confocal microscope with the Zen program (Zeiss).

\section{Flow cytometry analyses}

C20 cells stably expressing ER_K20 were seeded in a 6-well plate, and $24 \mathrm{~h}$ later the cells were treated with Brefeldin $A(10 \mu \mathrm{g} / \mathrm{mL})$ or Bafilomycin A1 (100 nM) or DMSO (as control) for $15 \mathrm{~h}$ at $37^{\circ} \mathrm{C}$. The cells were then harvested, washed in PBS and filtered with polystyrene tube with cell-strainer cap. The fluorescence of total 20,000 cells were recorded using the Aria II FACS system (BD Biosciences), and the data was processed with FlowJo. To measure ER_K20 fluorescence in siRNA-treated cells, C20 cells were transfected with siRNAs for 2 days before being analyzed by the flow cytometry.

\section{Cell fractionation and sucrose gradient ultracentrifugation}

To characterize subcellular localization of UFMylated targets, two $15-\mathrm{cm}$ dishes of WT or UFSP2 knockout HEK293T cells were harvested and washed in ice-cold PBS. Cells were resuspended in $3 \mathrm{~mL}$ hypotonic buffer (50 mM HEPES, pH 7.3, $10 \mathrm{mM} \mathrm{KCl}$ and 2 $\mathrm{mM} \mathrm{MgCl}$ ) containing EDTA-free Protease Inhibitor Cocktail and incubated on ice for $5 \mathrm{~min}$ before being homogenized by a Dounce homogenizer. Sucrose was added to final concentration of $250 \mathrm{mM}$ to preserve integrity of subcellular organelles and membranes. Cell homogenates were subjected to centrifugation at $1,000 \times g$ for $5 \mathrm{~min}$ to remove unbroken cells and nuclei. The supernatant was then ultracentrifuged at $100,000 \times g$ for 20 min at $4{ }^{\circ} \mathrm{C}$, resulting in a cytosol-containing supernatant fraction and a membrane pellet. The membrane pellet was washed once with 1 $\mathrm{mL}$ phosphate buffer saline (PBS) and resuspended in $300 \mu \mathrm{L}$ membrane resuspension buffer (50 mM HEPES, pH 7.3, 1 mM DTT) with EDTA-free Protease Inhibitor Cocktail. A fraction of the membrane and cytosol was analyzed by immunoblotting. The remaining membrane fraction was layered on top of a gradient sucrose cushion (15\%-45\%) and centrifuged at $100,000 \times g$ for $12 \mathrm{~h}$ at $4{ }^{\circ} \mathrm{C}$. Fractions of equal volume were collected for immunoblotting analyses. To characterize biochemically the localization of ERtargeted ribosome stalling reporters, $0.5 \times 10^{6} \mathrm{HEK} 293 \mathrm{~T}$ cells were seeded in each well of a 6-well plate. After $24 \mathrm{~h}$, cells in each well were transfected with $2 \mu \mathrm{g}$ reporter plasmids either by itself or together with $0.2 \mu \mathrm{g}$ UFM1-expressing plasmids (for rescue) or empty vector. After $30 \mathrm{~h}$, cells were collected, washed in ice-cold PBS and resuspended in $1 \mathrm{~mL}$ hypotonic buffer $(20 \mathrm{mM}$ HEPES, $\mathrm{pH}$ $7.3,5 \mathrm{mM} \mathrm{Mg}(\mathrm{OAc})_{2}$ ) containing $50 \mu \mathrm{g} / \mathrm{mL}$ cycloheximide, $20 \mathrm{U} / \mathrm{mL}$ SUPERase In RNase Inhibitor, $1 \mathrm{mM}$ DTT and EDTA-free Protease Inhibitor Cocktail, and incubated on ice for $5 \mathrm{~min}$ before being homogenized by a Dounce homogenizer. Cell extracts were centrifuged at $1,000 \times g$ for $5 \mathrm{~min}$ to remove nuclei. The supernatant was then centrifuged at $14,000 \times \mathrm{g}$ for $15 \mathrm{~min}$ at $4{ }^{\circ} \mathrm{C}$ to pellet the membranes. The resulting supernatant was recovered as cytosol fraction, whereas the membrane pellet was washed once with $1 \mathrm{~mL}$ hypotonic buffer and centrifuged again at $14,000 \times g$ for $10 \mathrm{~min}$. The membrane pellet was re-suspended in the Laemmli sample buffer and boiled at $95^{\circ} \mathrm{C}$ for immunoblotting analysis.

\section{Polysome profiling}

Polysome profiling were performed as previously described. ${ }^{57}$ Briefly, $8 \times 10^{6}$ HEK293T cells treated with anisomycin or DMSO were lysed in the polysome buffer $(25 \mathrm{mM}$ Tris- $\mathrm{HCl}, \mathrm{pH} 7.4,150$ $\mathrm{mM} \mathrm{NaCl}, 15 \mathrm{mM} \mathrm{MgCl} 2,1 \mathrm{mM}$ DTT, $1 \%$ Triton X-100, $100 \mathrm{mg} / \mathrm{mL}$ cycloheximide, $200 \mathrm{U} / \mathrm{mL}$ SUPERase In RNase inhibitor, $20 \mathrm{U} / \mathrm{mL}$ Turbo DNase and complete EDTA-free protease inhibitor cocktail) on ice for $20 \mathrm{~min}$. After lysis, nuclei were removed by two consecutive centrifugations at $1,800 \times g$ for $5 \mathrm{~min}$ at $4{ }^{\circ} \mathrm{C}$ followed by one centrifugation at $12,000 \times g$ for $10 \mathrm{~min}$. RNA concentrations were measured using Nanodrop UV spectrophotometer (Thermo Fisher Scientific) and normalized RNA $(600 \mu \mathrm{g})$ was layered onto a linear sucrose gradient $(10 \%-50 \%$ sucrose $(\mathrm{w} / \mathrm{v}), 25 \mathrm{mM}$ Tris- $\mathrm{HCl}$, $\mathrm{pH}$ 7.4, $150 \mathrm{mM} \mathrm{NaCl}, 15 \mathrm{mM} \mathrm{MgCl}, 1 \mathrm{mM} \mathrm{DTT}, 100 \mu \mathrm{g} / \mathrm{mL}$ cycloheximide) and centrifuged in a SW41 Ti rotor (Beckman) for 3 h at $40,000 \mathrm{rpm}$ at $4{ }^{\circ} \mathrm{C}$. Fractions $(0.5 \mathrm{~mL})$ were collected using the Density Gradient Fraction System (Brandel) while the OD254 was continuously recorded. Fraction samples were analyzed by SDSPAGE followed by immunoblotting.

\section{Immunoblotting}

Immunoblotting was performed using standard protocols. Proteins were separated in NuPAGE (4\%-12\%) Bis-Tris gels (Invitrogen) and transferred onto nitrocellulose membranes (BioRad). Target protein was detected by specific primary antibodies followed by secondary horseradish peroxidase (HRP)-conjugated antibodies (for less abundant proteins) (Sigma) or fluorescently labeled antibodies (for abundant antigens) (Invitrogen). For immunoblotting with HRP-conjugated secondary antibodies, signal was detected by the enhanced chemiluminescence method (ECL) using the Immobilon western chemiluminescent HRP substrate (Millipore) and recorded by a Fuji LAS-4000 imager. The intensity of the detected protein bands was quantified by ImageGauge v3.0 software. For immunoblotting with fluorescently labeled secondary antibodies, membranes were scanned using a LI-COR Odyssey scanner and intensity of protein bands was quantified by the Odyssey software.

\section{Ribosome fractionation}

Ribosomes were purified as previously described. ${ }^{58}$ Briefly, HEK293T cells from two wells of a 6 -well plate ( $\sim$ million cells) were collected and washed with ice-cold PBS. Cells were then lysed in $300 \mu \mathrm{L}$ buffer A ( $50 \mathrm{mM}$ Tris- $\mathrm{HCl}$ pH 7.4, $250 \mathrm{mM} \mathrm{KCl}, 5 \mathrm{mM}$ $\mathrm{MgCl}_{2}, 250 \mathrm{mM}$ Sucrose, $0.7 \%$ NP40) with an EDTA-free protease inhibitor cocktail on ice for $15 \mathrm{~min}$. After lysis, cells were centrifuged by two consecutive centrifugations, first at $750 \times g$ for $5 \mathrm{~min}$ followed by $12,500 \times \mathrm{g}$ for $10 \mathrm{~min}$ at $4{ }^{\circ} \mathrm{C}$ to remove nuclei and mitochondria. Supernatant was collected and $\mathrm{KCl}$ was added to a final concentration of $0.5 \mathrm{M}$. Cell extracts were layered on top of $0.8 \mathrm{~mL}$ sucrose cushion $(50 \mathrm{mM}$ Tris- $\mathrm{HCl} \mathrm{pH} \mathrm{7.4,500} \mathrm{mM}$ $\mathrm{KCl}, 5 \mathrm{mM} \mathrm{MgCl} 2$ and $1 \mathrm{M}$ sucrose) and centrifuged at 250,000× for $2.5 \mathrm{~h}$ at $4{ }^{\circ} \mathrm{C}$. After the supernatant (ribosome-free fraction) was removed, the ribosome pellet was washed once with ultrapure water and re-suspended in Laemmli sample buffer and boiled at $95^{\circ} \mathrm{C}$ for immunoblotting analysis. To detect the association of nascent polypeptides with ribosomes, 3.5 million HEK293T cells were seeded in a $10-\mathrm{cm}$ dish. After $24 \mathrm{~h}$, cells were transfected with $5 \mu \mathrm{g}$ plasmids expressing either ER_K20 or ER_K0. At $24 \mathrm{~h}$ post-transfection, 4 million cells were harvested, radioactively labeled with $4 \mathrm{mCi}\left[{ }^{35} \mathrm{~S}\right.$-methionine/cysteine for $30 \mathrm{~min}$ at $37^{\circ} \mathrm{C}$ 
and then immediately lysed in $300 \mu \mathrm{L}$ polysome buffer for $20 \mathrm{~min}$ on ice. After centrifugation at $14,000 \times g$ for $5 \mathrm{~min}$ at $4{ }^{\circ} \mathrm{C}$, the cleared cell lysate was layered on top of $0.8 \mathrm{~mL}$ sucrose cushion (supplemented with $20 \mathrm{U} / \mathrm{mL}$ SUPERase In RNase inhibitor) and centrifuged at $250,000 \times g$ for $2.5 \mathrm{~h}$ at $4{ }^{\circ} \mathrm{C}$. The resulting supernatant (the ribosome-free fraction) was carefully transferred to a new tube and SDS was added at a final concentration of $0.1 \%$. The ribosome pellet was washed once with ultrapure water and completely dissolved in $500 \mu \mathrm{L}$ RIPA buffer supplemented with 1 mM DTT and an EDTA-free protease inhibitor cocktail. Both ribosome and ribosome-free fractions were subjected to immunoprecipitation using anti-FLAG $\mathrm{M} 2$ beads at $4{ }^{\circ} \mathrm{C}$ for $1 \mathrm{~h}$. The beads were washed twice with NET buffer containing $0.1 \%$ SDS and boiled in $36 \mu \mathrm{L}$ Laemmli sample buffer. The eluted proteins were resolved in Criterion ${ }^{\mathrm{TM}} \mathrm{TGX}^{\mathrm{TM}}$ precast gels (10\%) followed by autoradiography analyses.

Proteinase $\mathrm{K}$ protection assay and Endo $\mathrm{H}$ treatment Three million HEK293T cells were seeded in a $10-\mathrm{cm}$ dish and transfected with $5 \mu \mathrm{g}$ ER_K20 plasmids $24 \mathrm{~h}$ later. Four million cells were harvested at $24 \mathrm{~h}$ post-transfection and incubated in $2 \mathrm{~mL}$ DMEM medium free of methionine and cysteine (Starvation medium) at $37^{\circ} \mathrm{C}$ for $40 \mathrm{~min}$. Cells were labeled with $4 \mathrm{mCi}\left[{ }^{35} \mathrm{~S}\right]-$ methionine/cysteine for $30 \mathrm{~min}$ at $37^{\circ} \mathrm{C}$. After centrifugation and removal of labeling medium, cells were permeabilized in $150 \mu \mathrm{L}$ PB buffer containing $0.025 \%$ digitonin (no protease inhibitor) on ice for $5 \mathrm{~min}$, followed by centrifugation at $14,000 \times g$ for $5 \mathrm{~min}$ at $4{ }^{\circ} \mathrm{C}$. After the supernatant (cytosol) was removed, the pelleted membranes were washed one time with PB buffer without digitonin. Washed membranes were resuspended in $150 \mu \mathrm{L} P B$ buffer and the sample was divided into three equal portions $(A, B$, C, $40 \mu \mathrm{L}$ each). To portion C, $5 \mu \mathrm{L}$ of $10 \%$ NP40 was added, while to portion $A$ and $B, 5 \mu \mathrm{L}$ water was added. Proteinase $K$ diluted with the PB buffer was then added at $4 \mu \mathrm{g} / \mathrm{mL}$ to portion $B$ and $C$. The membranes were incubated on ice for $30 \mathrm{~min}$. After treatment, the membrane was pelleted, and washed with PB buffer (with complete protease inhibitor cocktail and $1 \mathrm{mM}$ DTT) and solubilized in $500 \mu \mathrm{L}$ of RIPA buffer. Centrifugation cleared membrane extracts were subjected to immunoprecipitation with anti-FLAG M2 beads. For Endo $\mathrm{H}$ treatment, samples in Laemmli buffer were diluted 4-fold with ultrapure water, heat-denatured. Forty microliters samples were incubated with $5 \mu \mathrm{L} 10 \times \mathrm{G} 5$ buffer (NEB) plus 500 units of Endo $\mathrm{H}$ (NEB) at $37^{\circ} \mathrm{C}$ for $1 \mathrm{~h}$. The reactions were stopped by addition of $4 \times$ Laemmli buffer and boiling. Samples were analyzed by SDS-PAGE and autoradiography.

\section{Generation of CRISPR knockout cells}

CRISPR knockout (KO) cell lines were generated using Cas9 D10A nickase together with two sgRNAs, which has been reported to greatly reduce off-target editing. ${ }^{55}$ One million HEK293T or K562 cells were co-transfected with pX330 vectors containing distinct sgRNAs ( $2 \mu \mathrm{g}$ plasmid each). Cells were single-cell seeded into 96wells at $48 \mathrm{~h}$ post-transfection. Clones derived from single cells were harvested and screened for successful gene knockout by immunoblotting.

\section{Secretion assay}

To measure the secretion of endogenous Clusterin, 0.7 million $\mathrm{K} 562$ cells were treated with $50 \mu \mathrm{M}$ hemin in $1 \mathrm{~mL}$ fresh medium for $24 \mathrm{~h}$. After treatment, cells were pelleted at $500 \times \mathrm{g}$ for $3 \mathrm{~min}$ and washed once with pre-warmed medium. Cells were then resuspended in $1 \mathrm{~mL}$ culture medium, seeded in a 12-well plate and incubated at $37{ }^{\circ} \mathrm{C}$ with $5 \% \mathrm{CO}_{2}$ in the incubator. At each time point, the plate was centrifuged at $1,000 \times g$ for $1 \mathrm{~min}$ to sediment cells. Conditioned medium $(150 \mu \mathrm{L})$ were collected and centrifuged at $10,000 \times g$ for $10 \mathrm{~min}$ at $4{ }^{\circ} \mathrm{C}$. Ninety microliters of cleared medium supernatant was mixed with $30 \mu \mathrm{L}$ of $4 \times$ Laemmli sample buffer and boiled at $95^{\circ} \mathrm{C}$ for $10 \mathrm{~min}$. Cells were collected at the end of secretion assay and directly dissolved in Laemmli sample buffer and boiled at $95^{\circ} \mathrm{C}$ for $20 \mathrm{~min}$.

For ER_GFP secretion assay, 1 million $\mathrm{K} 562$ cells were electroporated with $5 \mu \mathrm{g}$ ER_GFP-expressing plasmid using the Nucleofector 2b system following manufacturer's instructions. After $24 \mathrm{~h}$, cells were collected, washed once with pre-warmed medium and treated with $50 \mu \mathrm{M}$ hemin for another $24 \mathrm{~h}$. After treatment, cells were centrifuged at $500 \times g$ for $3 \mathrm{~min}$ and overnight medium was removed. Cells were then washed once with pre-warmed medium, resuspended in $1 \mathrm{~mL}$ fresh medium and incubated at $37^{\circ} \mathrm{C}$. At each time point, cells were pelleted and $450 \mu \mathrm{L}$ conditioned medium was collected and centrifuged at $1,000 \times g$ for $3 \mathrm{~min}$ and then at $10,000 \times g$ for $8 \mathrm{~min}$ at $4{ }^{\circ} \mathrm{C}$. For immunoprecipitation of secreted ER_GFP, $350 \mu \mathrm{L}$ cleared conditioned medium was incubated with an anti-GFP antibody for 30 min at $4^{\circ} \mathrm{C}$, followed by incubation with $40 \mu \mathrm{L}$ slurry of protein $\mathrm{A}$ beads for $1 \mathrm{~h}$ at $4^{\circ} \mathrm{C}$. Beads were washed once with ice-cold PBS and bound proteins were dissolved by boiling beads in $30 \mu \mathrm{L}$ Laemmli sample buffer at $95^{\circ} \mathrm{C}$ for $5 \mathrm{~min}$. Cells were collected at the end of secretion assay and directly dissolved in Laemmli sample buffer.

Reverse transcription quantitative PCR (qRT-PCR)

Total RNA was extracted from cells using TriPure reagent (Roche) and RNA was purified using RNeasy MinElute Cleanup Kit (Promega) following standard protocols. RNA concentration was measured by Nanodrop UV spectrophotometer and $500 \mathrm{ng}$ RNA was converted to CDNA using the iScript Reverse Transcription Supermix (BioRad) system. cDNA was diluted 2-fold and $1 \mu \mathrm{L}$ was used to perform qPCR using SsoAdvanced SYBR Green supermix kit (BioRad) on a CFX96 machine (BioRad). Data was analyzed using BioRad CFX manager 3.0 software. Ribosomal 18S RNA was used as a reference gene for quantification of gene expression. The following primers were used for qPCR:

SRPRa: forward, 5'-AGTTGGAGTGGTGTTTGTG-3'; reverse, 5'GCTCTCCTCTGCTTCACGAA-3'.

SRPRß: forward, 5'-GAGGCTTCAGTTCTTAGAGCGG-3'; reverse, 5'GGACTTGATACAGAAACTCAGCC-3'.

SEC61a: forward, 5'-CTCGCTTCAGTGGCAACTTGCT-3'; reverse, 5'GCCACCAACTGGATAAGCACGT-3'.

SEC61 $\beta$ : forward, 5'-CCGCACAACCTCGGCAGGCA-3'; reverse, 5'CAGAAGCGATGAACAGAAGACTC-3'.

\section{Ribosome-engaged transcriptome analysis}

Ninety million K562 cells were treated with or without hemin $(50 \mu \mathrm{M})$ for $24 \mathrm{~h}$. After treatment, 110 million cells were harvested for each condition and washed once with $20 \mathrm{~mL}$ ice-cold PBS. Cell pellets were immediately flash-frozen in liquid nitrogen. The cells were then resuspended in $1.5 \mathrm{~mL}$ polysome buffer and homogenized by passing through a $27 \mathrm{G} 1 \frac{1}{2}$ needle 10 times on ice. Trypan blue staining was used to confirm complete homogenization of the cell. The resulting cell lysates were then subjected to two consecutive centrifugations first at $1,800 \times g$ for $5 \mathrm{~min}$ and then at $12,500 \times \mathrm{g}$ for $10 \mathrm{~min}$ at $4{ }^{\circ} \mathrm{C}$ to remove nuclei and mitochondria, respectively. To pellet ribosomes, the cleared lysates were layered onto a $2 \mathrm{~mL}$ sucrose cushion (1 M sucrose, $20 \mathrm{mM}$ Tris, pH 7.4, $150 \mathrm{mM} \mathrm{NaCl}, 5 \mathrm{mM} \mathrm{MgCl}, 100 \mu \mathrm{g} / \mathrm{mL}$ cycloheximide, $20 \mathrm{U} / \mathrm{mL}$ SUPERase In RNase inhibitor, $1 \mathrm{mM}$ DTT and complete EDTA-free protease inhibitor) and centrifuged using a TLA100.4 rotor at $80,000 \mathrm{rpm}(346,716 \times \mathrm{g})$ for $4 \mathrm{~h}$ at $4{ }^{\circ} \mathrm{C}$. The ribosome pellet was washed twice with ice-cold ultrapure water and then dissolved in $1.5 \mathrm{~mL}$ polysome buffer followed by centrifugation at $10,000 \times g$ for 2 min at $4{ }^{\circ} \mathrm{C}$ to pellet any insoluble ribosome particles. The cleared supernatant containing total ribosomes was used for RNA extraction using Tripure reagents (Roche) and the RNeasy MinElute Cleanup Kit (Qiagen). RNA quality was evaluated by an Agilent bioanalyzer. RNA-seq was performed by the DNA Sequencing and Genomics Core at the 
National Heart, Lung, and Blood Institute (NHLBI) at NIH. RNA sequencing library was prepared using Illumina Ribo-Zero rRNA Removal Kit $(\mathrm{H} / \mathrm{M} / \mathrm{R})$ and deep sequencing was performed on the Illumina HiSeq2500 system to obtain single-end 75-bp reads. DNA sequences were trimmed of adapter sequences, assembled and aligned to human genome based on TopHat program. Reads Per Kilobase Million (RPKM) was calculated for transcript quantification. Gene enrichment analyses were performed using DAVID Bioinformatics Resources 6.8 (https://david.ncifcrf.gov/).

\section{Radiolabeling pulse-chase assay}

For radiolabeling pulse-chase assay, 3.5 million HEK293T cells were seeded in a $10-\mathrm{cm}$ dish. After $24 \mathrm{~h}$, cells were transfected with $5 \mu \mathrm{g}$ ER_K20-expressing plasmid and radiolabeling pulse-chase experiments were performed at $24 \mathrm{~h}$ post-transfection. For radiolabeling, 4 million cells were starved in $2 \mathrm{~mL}$ starvation medium (DMEM medium lacking cysteine and methionine, supplemented with $10 \%$ FBS) for $30 \mathrm{~min}$ at $37^{\circ} \mathrm{C}$, followed by labeling with $4 \mathrm{mCi}\left[{ }^{35} \mathrm{~S}\right]-$ methionine/cysteine in $300 \mu \mathrm{L}$ starvation medium for $16 \mathrm{~min}$ at $37^{\circ}$ C. MG132 and Brefeldin A were added during starvation, and chloroquine was added during radiolabeling. After labeling, cells were resuspended in $300 \mu \mathrm{L}$ starvation medium supplemented with $2.5 \mathrm{mM}$ methionine and cysteine. An aliquot of cells $(65 \mu \mathrm{L})$ was immediately taken out and mixed with $500 \mu \mathrm{L}$ RIPA buffer with 1 $\mathrm{mM}$ DTT and protease inhibitors. The remaining cells were incubated at $37^{\circ} \mathrm{C}$ and chased for 20, 40, and $60 \mathrm{~min}$. Equal amounts of cells $(65 \mu \mathrm{L})$ were taken at each time points and lysed in RIPA buffer. After centrifugation of cell lysate at $14,000 \times g$ for $5 \mathrm{~min}$, the cleared cell extracts were subjected to immunoprecipitation using anti-FLAG M2 beads. The beads were then washed twice with NET buffer with $0.1 \%$ SDS and bound proteins were eluted in $36 \mu \mathrm{L}$ Laemmli sample buffer by boiling at $95^{\circ} \mathrm{C}$ for $5 \mathrm{~min}$. Eluted proteins were analyzed by the SDS-PAGE using Criterion ${ }^{\mathrm{TM}} \mathrm{TGX}^{\mathrm{TM}}$ precast gels (10\%) followed by autoradiography analyses.

In vitro erythroid differentiation of human hematopoietic stem and progenitor cells (HSPCs)

Human HSPCs were prepared from peripheral blood by gradient centrifugation with Ficoll-Hypaque, washed and isolated using $\mathrm{CD}_{3}{ }^{+}$immunomagnetic beads (Miltenyi). Cells were cultured using a 2-phase approach consisting of 5 days in medium without erythropoietin (EPO) followed by a second phase with $1 \mathrm{U} / \mathrm{mL} E P O$, $1 \mu \mathrm{M}$ dexamethasone, $10 \mu \mathrm{M} \quad \beta$-mercaptoethanol, $0.3 \mathrm{mg} / \mathrm{mL}$ human holo-transferrin and $10 \mathrm{ng} / \mathrm{mL}$ stem cell factor for up to 12 days as previously described. ${ }^{59,60}$ Cells were collected at different days during the EPO-induced erythroid differentiation phase. Whole cell extracts were prepared by directly boiling cells in the Laemmli sample buffer and analyzed by immunoblotting assays.

Statistics and software

All experiments were repeated at least two times with representative gels shown. Unless specified, the $n$ values in the figure legend indicate the number of independent biological replicates. The number of repeats is also indicated by dots in column plots. Error bars were shown as mean \pm SEM, calculated by GraphPad Prism 7, and $P$-values were calculated by the GraphPad Prism 7 or by Student's $t$ test, as specified in the figure legends. Graphs were prepared with either GraphPad Prism 7 or Rstudio.

\section{ACKNOWLEDGEMENTS}

We thank Taplin mass spectrometry facility (Harvard Medical School) for protein identification service, NHLBI genomic core for RNA sequencing, W. Chen (NIDDK, NIH) for RNA sequence analysis, T. Rapoport for Sec61 $\beta$ antibodies, R. Hegde for NEMF antibodies, and the members of the Ye laboratory for discussions. L.W., Y.X., L.S., H.R., Y.Y., C.T.N., and N.R.G. are supported by an intramural research program of NIDDK in the National Institutes of Health, J.W.Y. is supported by an intramural research program of NIAID in the National Institutes of Health, H.L. is supported by NIH RO1DK113409.

\section{AUTHOR CONTRIBUTIONS}

L.W., X.Y., and Y.Y. designed the research. L.W., X.Y., L.S., and Y.Y. performed experiments. H.L. and J.W.Y. provided essential reagents and helped confirm RPL26 as a UFM1 substrate, N.R.G. contributed to the idea that ribosome stalling triggers UFMylation. H.R. and C.T.N. provides HSPCS. L.W. and Y.Y. wrote the paper and all authors participated in editing the manuscript.

\section{ADDITIONAL INFORMATION}

Supplementary information accompanies this paper at https://doi.org/10.1038/ s41422-019-0236-6.

Competing interests: The authors declare no competing interests.

\section{REFERENCES}

1. Christianson, J. C. \& Ye, Y. Cleaning up in the endoplasmic reticulum: ubiquitin in charge. Nat. Struct. Mol. Biol. 21, 325-335 (2014).

2. Ruggiano, A., Foresti, O. \& Carvalho, P. Quality control: ER-associated degradation: protein quality control and beyond. J. Cell Biol. 204, 869-879 (2014).

3. Needham, P. G., Guerriero, C. J. \& Brodsky, J. L. Chaperoning endoplasmic reticulum-associated degradation (ERAD) and protein conformational diseases. Cold Spring Harb. Perspect. Biol. 11, a033928 (2019).

4. Klauer, A. A. \& van Hoof, A. Degradation of mRNAs that lack a stop codon: a decade of nonstop progress. Wiley Interdiscip. Rev. RNA 3, 649-660 (2012).

5. Brandman, O. \& Hegde, R. S. Ribosome-associated protein quality control. Nat. Struct. Mol. Biol. 23, 7-15 (2016).

6. Joazeiro, C. A. P. Ribosomal stalling during translation: providing substrates for ribosome-associated protein quality control. Annu Rev. Cell Dev. Biol. 33, 343-368 (2017).

7. Brandman, O. et al. A ribosome-bound quality control complex triggers degradation of nascent peptides and signals translation stress. Cell 151, 1042-1054 (2012).

8. Juszkiewicz, S. \& Hegde, R. S. Initiation of quality control during Poly(A) translation requires site-specific ribosome ubiquitination. Mol. Cell 65, 743-750 e744 (2017).

9. Matsuo, Y. et al. Ubiquitination of stalled ribosome triggers ribosome-associated quality control. Nat. Commun. 8, 159 (2017).

10. Sundaramoorthy, E. et al. ZNF598 and RACK1 regulate mammalian ribosomeassociated quality control function by mediating regulatory $40 \mathrm{~S}$ ribosomal ubiquitylation. Mol. Cell 65, 751-760 e754 (2017).

11. Shao, S., von der Malsburg, K. \& Hegde, R. S. Listerin-dependent nascent protein ubiquitination relies on ribosome subunit dissociation. Mol. Cell 50, 637-648 (2013).

12. Juszkiewicz, S. et al. ZNF598 is a quality control sensor of collided ribosomes. Mol. Cell 72, 469-481 e467 (2018).

13. Shao, S. \& Hegde, R. S. Reconstitution of a minimal ribosome-associated ubiquitination pathway with purified factors. Mol. Cell 55, 880-890 (2014).

14. Bengtson, M. H. \& Joazeiro, C. A. Role of a ribosome-associated E3 ubiquitin ligase in protein quality control. Nature 467, 470-473 (2010).

15. Yonashiro, R. et al. The Rqc2/Tae2 subunit of the ribosome-associated quality control (RQC) complex marks ribosome-stalled nascent polypeptide chains for aggregation. Elife 5, e11794 (2016).

16. Shao, S., Brown, A., Santhanam, B. \& Hegde, R. S. Structure and assembly pathway of the ribosome quality control complex. Mol. Cell 57, 433-444 (2015).

17. von der Malsburg, K., Shao, S. \& Hegde, R. S. The ribosome quality control pathway can access nascent polypeptides stalled at the Sec61 translocon. Mol. Biol. Cell 26, 2168-2180 (2015).

18. Choe, Y. J. et al. Failure of RQC machinery causes protein aggregation and proteotoxic stress. Nature 531, 191-195 (2016).

19. Chu, J. et al. A mouse forward genetics screen identifies LISTERIN as an E3 ubiquitin ligase involved in neurodegeneration. Proc. Natl Acad. Sci. USA 106, 2097-2103 (2009).

20. Shen, P. S. et al. Protein synthesis. Rqc $2 p$ and 60 S ribosomal subunits mediate mRNA-independent elongation of nascent chains. Science 347, 75-78 (2015).

21. Liakath-Ali, K. et al. An evolutionarily conserved ribosome-rescue pathway maintains epidermal homeostasis. Nature 556, 376-380 (2018).

22. Ishimura, R. et al. RNA function. Ribosome stalling induced by mutation of a CNSspecific tRNA causes neurodegeneration. Science 345, 455-459 (2014).

23. Komatsu, M. et al. A novel protein-conjugating system for Ufm1, a ubiquitin-fold modifier. EMBO J. 23, 1977-1986 (2004). 
24. Cai, Y. et al. UFBP1, a key component of the Ufm 1 conjugation system, is essential for ufmylation-mediated regulation of erythroid development. PLoS Genet. 11, e1005643 (2015).

25. Tatsumi, K. et al. The Ufm1-activating enzyme Uba5 is indispensable for erythroid differentiation in mice. Nat. Commun. 2, 181 (2011).

26. Zhang, M. et al. RCAD/Ufl1, a Ufm1 E3 ligase, is essential for hematopoietic stem cell function and murine hematopoiesis. Cell Death Differ. 22, 1922-1934 (2015).

27. Nahorski, M. S. et al. Biallelic UFM1 and UFC1 mutations expand the essential role of ufmylation in brain development. Brain 141, 1934-1945 (2018).

28. Muona, M. et al. Biallelic variants in UBA5 link dysfunctional UFM1 ubiquitin-like modifier pathway to severe infantile-onset encephalopathy. Am. J. Hum. Genet. 99, 683-694 (2016).

29. Colin, E. et al. Biallelic variants in UBA5 reveal that disruption of the UFM1 cascade can result in early-onset encephalopathy. Am. J. Hum. Genet. 99, 695-703 (2016).

30. Duan, R. et al. UBA5 mutations cause a new form of autosomal recessive cerebellar ataxia. PLOS ONE 11, e0149039 (2016).

31. Yoo, H. M. et al. Modification of ASC1 by UFM1 is crucial for ERalpha transactivation and breast cancer development. Mol. Cell 56, 261-274 (2014).

32. Liu, J. et al. A critical role of DDRGK1 in endoplasmic reticulum homoeostasis via regulation of IRE1alpha stability. Nat. Commun. 8, 14186 (2017).

33. Qin, B. et al. UFL1 promotes histone $\mathrm{H} 4$ ufmylation and ATM activation. Nat. Commun. 10, 1242 (2019).

34. Simsek, D. et al. The mammalian ribo-interactome reveals ribosome functional diversity and heterogeneity. Cell 169, 1051-1065 e1018 (2017).

35. Walczak, C. P. et al. Ribosomal protein RPL26 is the principal target of UFMylation. Proc. Natl Acad. Sci. USA 116, 1299-1308 (2019).

36. Tatsumi, K. et al. A novel type of $E 3$ ligase for the Ufm1 conjugation system. J. Biol. Chem. 285, 5417-5427 (2010).

37. Kang, S. H. et al. Two novel ubiquitin-fold modifier 1 (Ufm1)-specific proteases, UfSP1 and UfSP2. J. Biol. Chem. 282, 5256-5262 (2007).

38. Lemaire, K. et al. Ubiquitin fold modifier 1 (UFM1) and its target UFBP1 protect pancreatic beta cells from ER stress-induced apoptosis. PLOS ONE 6, e18517 (2011).

39. Garreau de Loubresse, N. et al. Structural basis for the inhibition of the eukaryotic ribosome. Nature 513, 517-522 (2014).

40. Ingolia, N. T., Lareau, L. F. \& Weissman, J. S. Ribosome profiling of mouse embryonic stem cells reveals the complexity and dynamics of mammalian proteomes. Cell 147, 789-802 (2011).

41. Pestka, S. Inhibitors of ribosome functions. Annu. Rev. Microbiol 25, 487-562 (1971).

42. Dimitrova, L. N., Kuroha, K., Tatematsu, T. \& Inada, T. Nascent peptide-dependent translation arrest leads to Not4p-mediated protein degradation by the proteasome. J. Biol. Chem. 284, 10343-10352 (2009).

43. Verma, R. et al. Vms1 and ANKZF1 peptidyl-tRNA hydrolases release nascent chains from stalled ribosomes. Nature 557, 446-451 (2018).
44. Rubinsztein, D. C. Cell biology: receptors for selective recycling. Nature 522, 291-292 (2015)

45. Wyant, G. A. et al. NUFIP1 is a ribosome receptor for starvation-induced ribophagy. Science 360, 751-758 (2018).

46. Andersson, L. C., Jokinen, M. \& Gahmberg, C. G. Induction of erythroid differentiation in the human leukaemia cell line K562. Nature 278, 364-365 (1979).

47. An, X. et al. Global transcriptome analyses of human and murine terminal erythroid differentiation. Blood 123, 3466-3477 (2014).

48. $\mathrm{Hu}, \mathrm{X}$. et al. Ubiquitin-fold modifier 1 inhibits apoptosis by suppressing the endoplasmic reticulum stress response in Raw264.7 cells. Int. J. Mol. Med. 33, 1539-1546 (2014)

49. Zhang, Y., Zhang, M., Wu, J., Lei, G. \& Li, H. Transcriptional regulation of the Ufm 1 conjugation system in response to disturbance of the endoplasmic reticulum homeostasis and inhibition of vesicle trafficking. PLOS ONE 7, e48587 (2012).

50. Crowder, J. J. et al. Rkr1/Ltn1 ubiquitin ligase-mediated degradation of translationally stalled endoplasmic reticulum proteins. J. Biol. Chem. 290, 18454-18466 (2015).

51. Ast, T., Michaelis, S. \& Schuldiner, M. The protease Ste24 clears clogged translocons. Cell 164, 103-114 (2016).

52. Kuroha, K., Zinoviev, A., Hellen, C. U. T. \& Pestova, T. V. Release of ubiquitinated and non-ubiquitinated nascent chains from stalled mammalian ribosomal complexes by ANKZF1 and Ptrh1. Mol. Cell 72, 286-302 e288 (2018).

53. Zurita Rendon, O. et al. Vms1p is a release factor for the ribosome-associated quality control complex. Nat. Commun. 9, 2197 (2018).

54. Mills, E. W., Wangen, J., Green, R. \& Ingolia, N. T. Dynamic regulation of a ribosome rescue pathway in erythroid cells and platelets. Cell Rep. 17, 1-10 (2016).

55. Ran, F. A. et al. Double nicking by RNA-guided CRISPR Cas9 for enhanced genome editing specificity. Cell 154, 1380-1389 (2013).

56. Leonetti, M. D., Sekine, S., Kamiyama, D., Weissman, J. S. \& Huang, B. A scalable strategy for high-throughput GFP tagging of endogenous human proteins. Proc. Natl Acad. Sci. USA 113, E3501-E3508 (2016).

57. Guydosh, N. R. \& Green, R. Dom34 rescues ribosomes in 3' untranslated regions. Cell 156, 950-962 (2014).

58. Belin, S. et al. Purification of ribosomes from human cell lines. in Current Protocols in Cell Biology, Ch. 3, Unit 3 40, https://doi.org/10.1002/0471143030.cb0340s49 (2010).

59. Fibach, E. Techniques for studying stimulation of fetal hemoglobin production in human erythroid cultures. Hemoglobin 22, 445-458 (1998).

60. Vaisman, B., Meyron-Holtz, E. G., Fibach, E., Krichevsky, A. M. \& Konijn, A. M. Ferritin expression in maturing normal human erythroid precursors. Br. J. Haematol. 110, 394-401 (2000).

61. Myasnikov, A. G. et al. Structure-function insights reveal the human ribosome as a cancer target for antibiotics. Nat. Commun. 7, 12856 (2016).

62. Behrmann, E. et al. Structural snapshots of actively translating human ribosomes. Cell 161, 845-857 (2015). 